\title{
1 ASpediaFI: Functional interaction analysis of alternative splicing
}

2

3

4 Doyeong Yu*, Kyubin Lee*, Daejin Hyung, Soo Young Cho, and Charny Park ${ }^{\dagger}$

5 Bioinformatics Branch, Research Institute, National Cancer Center, 232 Ilsan-ro, Goyang-si,

6 Kyeonggi-do 10408, Republic of Korea

7

8 Doyeong Yu: nachoryu@ncc.re.kr

$9 \quad$ Kyubin Lee: rbqlsrbqls56@ncc.re.kr

10 Daejin Hyung: daejin0709@ncc.re.kr

11 Soo Young Cho: sooycho@ncc.re.kr

12 Charny Park: charn78@ ncc.re.kr

13

$14 *$ These authors contributed equally to this work as first authors.

$15 \dagger$ To whom correspondence should be addressed. Tel: +82-31-920-2581; Email:

16 charn78@ncc.re.kr, charn78@gmail.com

17 Present Address: Charny Park, Bioinformatics Team, Research Institute, National Cancer

18 Center, 323 Ilsanro Ilsandonggu Goyangsi, Gyeonggido 10408, Republic of Korea

19

\section{events}




\section{ABSTRACT}

21 Alternative splicing (AS) regulates biological process governing phenotype or disease.

22 However, it is challenging to systemically analyze global regulation of AS events, their gene

23 interactions, and functions. Here, we introduce a novel application, ASpediaFI for identifying

24 AS events and co-regulated gene interactions implicated in pathways. Our method establishes

25 an interaction network including AS events, performs random walk with restart, and finally

26 identifies a functional subnetwork containing the AS event. We validated the capability of

27 ASpediaFI to interpret biological relevance based on three case studies. Using simulation

28 data, we achieved higher accuracy than with other methods and detected pathway-associated

29 AS events.

\section{Keywords}

32 Alternative splicing, RNA-Seq, Gene set enrichment analysis, Random walk with restart, co-

33 expressed gene, Splicing factor, Gene interaction, Subnetwork identification 


\section{BACKGROUND}

36 Alternative splicing (AS) is a key regulatory mechanism that confers transcript diversity and

37 phenotypic plasticity in eukaryotes [1]. In normal cells, splicing factors induce tissue-specific

38 mRNA expression and embryonic stem cell differentiation [2,3]. In contrast, splice site

39 mutations or splicing factor (SF) variants reprogram global splicing events and induce

40 aberrant junctions in cancer cells and other diseased cells [4-6]. Aberrant AS events in cancer

41 cells disrupt the function of tumor suppressor genes and activate the oncogenic pathways [6].

42 Hundreds of RNA-binding proteins, the members of the spliceosome, play a regulatory role

43 in the cell; however, the functional effect of the spliceosome is not fully understood. As

44 several splicing events occur simultaneously, it is challenging to infer the effects of

45 cooperative regulation with genes and consensus pathway enrichment.

46 To identify the differential splicing and biological relevance, the fundamental strategy is

47 categorized as the exon- or isoform-level approaches. The exon-level approach calculates

48 percent spliced-in (PSI) values or total read counts from exon and junction read counts. The

49 counts indicate exon usage, which is used for testing differential AS (DAS) events. Accurate

50 statistical models have been developed to detect DAS that rank DAS events by significance

51 [7-10]. However, unlike various downstream methods for gene expression analysis, the AS

52 analysis method is restricted to inferring functional regulation induced by DAS events [11].

53 Previously developed application psichomics provides various downstream analyses,

54 including the correlation between DAS and gene expression for user convenience [11].

55 However, they do not identify the integrative co-regulation of AS for systematically

56 uncovering pathways. To reveal the splicing regulatory network, pCastNet identifies

57 associations between exon and upstream regulators or downstream target genes using partial

58 correlation. This approach requires a large number of samples (e.g. multiple tissues), and 
59 supports only the method without execution file. In spite of novel method development,

60 exon-level approaches are restricted in DAS and their results are difficult to interpret

61 genome-wide regulation and functions by splicing.

62 To uncover functional regulation, splicing studies using isoform expression also apply

63 differential expression test and establish co-regulation network. Differentially expressed

64 isoforms were tested like DEG test for each isoform [12]. Because isoform abundance is

65 estimated from whole gene region, the methods result stable expression profile and DEG [12].

66 However these also include other limitation. Even though, major isoform differentially

67 expressed in various conditions, isoform ratio within single gene could maintain. It is

68 irrelevant to identify switch-like exons to regulate critical function. Nevertheless, isoform

69 abundance is versatile to calculate expression correlations between gene pairs. To establish

70 tissue-specific transcriptome-wide networks (TWN), previous study considered both gene

71 and isoform expression. They identified switch-like isoforms to compute isoform ratio and

72 established tissue-specific TWN $[2,13]$. TWN successfully elucidated tissue-specific

73 molecular functions. While this method has the advantage of capturing post-transcriptional

74 interactions, it is not adequate for tracing genomic regions of spliced exons or functional

75 sequences like protein domains or NMD. Additionally, the isoform-level approach cannot

76 verify cis-element usages like a donor-acceptor site, or other motifs to recognize spliceosome

$77[6,14,15]$. Therefore, a novel integrative method is required to investigate AS events and their

78 functional interactions with partner genes as well as biological processes.

79 Recent studies have identified transcriptional regulation by the spliceosome in various

80 conditions, including cancer, embryonic development, and other cellular phenotypes

$81[3,5,6,16]$. To reveal the global regulation by SFs, studies aimed at the identification of

82 specific biological processes and the cooperative interactions were initiated $[3,13,17]$. 
83 Unfortunately, these approaches for identifying both splicing and associated pathways were

84 restricted to a simple GSEA method, and independent tests for both DAS and DEG sets were

85 performed $[5,6,13]$. Further, performance of multiple independent tests for splicing and gene

86 expression does not enable the inference of global regulation by spliceosome and the

87 interactions between AS and partner genes. Although the current gene set databases such as

88 hallmark or REACTOME are appropriate for testing enrichment derived from gene

89 expression $[18,19]$, these enrichment tests using known gene sets may fail to identify novel

90 splicing events and pertinent global interactions of the spliced genes.

91 Therefore, we developed a novel method ASpediaFI (Alternative Splicing Encyclopedia:

92 Functional Interaction) to systematically identify functional AS events correlated with genes

93 involved in pathways. We applied guilt-by-association generally used for gene expression

94 analysis to splicing regulation. In order to reveal transcriptome-wide global regulation of both

95 spliced genes and non-spliced genes, we established a heterogeneous interaction network for

96 both genes and AS events. To increase interpretation availability, pathways including gene

97 set information were also included to the network. Our applications explore splicing

98 subnetwork regulated by SF conditions using discriminative random walks with restart

99 (DRaWR). The algorithm has been applied to various heterogeneous networks like gene co-

100 expressed interactions, sequence homology, or transcription factor-binding motif [20,21].

101 Random walk with restart (RWR) algorithm explores the interaction networks from a query

102 gene set - called seed, and finally ranks nodes based on association with the query. To

103 confirm whether our analysis method produces a biologically relevant result, we applied our

104 method to three RNA-Seq datasets, which included samples from cancer patients with the SF

105 variant and SF knockdown cells. We compared our results for three RNA-Seq dataset with

106 previous results and other tools. The result was verified in various aspects like AS event types' 
107 proportion, biological relevance, discriminative power, and other parameters. We also

108 evaluated the performance of our method using simulated dataset. ASpediaFI is available in

109 Bioconductor (https://bioconductor.org/packages/ASpediaFI).

\section{RESULTS}

\section{ASpediaFI algorithm and analysis workflow}

113 ASpediaFI identified a subnetwork from a heterogeneous network established using gene-

114 gene interactions, containing gene-AS and gene-pathway interactions. The interaction

115 network was based on the concept of guilt by association, which states that associated or

116 interacting genes are more probable to share function [21]. We expanded the network by

117 adding AS events and pathways to the feature nodes. Quantitative information weighting

118 network edges were collected from PSI, gene expression and pathway gene sets. The

119 ASpediaFI workflow starts with data preparation and sequentially follows through

120 heterogeneous network establishment, subnetwork exploration, and further downstream

121 analysis. During the data preparation step, our method identifies AS events from gene model

122 annotation, collects gene expression, calculates PSI profile, and refers pathway gene sets and

123 gene interaction data collected from public databases (Figure 1A). ASpediaFI integrates the

124 processed data to construct a heterogeneous network that contains gene nodes and its feature

125 nodes representing AS event and pathway. Before executing the algorithm, the adjacency

126 network is normalized within the feature nodes and for all nodes. Next, to explore the

127 subnetworks, our method performs DRaWR on the heterogeneous network using previously

128 defined relevant query gene sets collected from DEGs (Figure 1B, blue node) [21]. In the first

129 stage, our algorithm explores the highly ranked feature nodes from the query set. We then 
130 extract a subnetwork from these feature nodes chosen from the first stage and all gene nodes,

131 including associated edges (Figure 1B). Next, ASpediaFI performs second stage RWR for

132 gene nodes to rank again and additionally calculates $P$-values by permutation tests to

133 eliminate background effects like query gene size. For user convenience, our tool provides

134 further analyses, including GSEA and data visualization. More details of our algorithm are

135 described in the Method section.

\section{Alternative splicing analysis using three RNA-Seq datasets applying ASpediaFI}

137 To verify the capability of ASpediaFI, we analyzed three RNA-Seq datasets representing the

138 following: myelodysplastic syndrome (MDS), stomach cancer (STAD), and RBFOX1-

139 knockdown cell lines. MDS and STAD were collected from cancer patients, and RBFOX1

140 has replicated samples of a relatively smaller size ( $n=5$ per condition). The datasets contain

141 SF mutations or down-regulations. We compared the SF deficiency profiles with the wild-

142 type using ASpediaFI and investigated whether our DAS sets determine the splicing pattern

143 and cis-element usage by the spliceosome. The biological relevance of our highly ranked

144 pathway result was delineated by referring to previous studies, and the consistency of GSEA

145 in using gene expression was also evaluated. Additionally, we tested how much our DAS set

146 was enriched in known and novel pathways or how much our result was coherent based on

147 other known AS signatures compared to other methods. In a further overall investigation of

148 the DAS set, we thoroughly examined the DAS events belonging to known and novel

149 pathways compared to other results. Each spliced gene was reviewed for biological relevance

150 and functional consistency with identified pathways. Additionally, functional sequence

151 features like protein domain and NMD that exist on spliced exons were extensively

152 investigated and compared with other results [22]. 
153 Case study 1: Three splicing factor mutations in myelodysplastic syndrome induce the 154 dysregulation of heme metabolism.

155 We investigated AS events in RNA-Seq samples from MDS patients $(n=84)$ with SF

156 deficiency on SF3B1 $(n=28), \operatorname{SRSF} 2(n=8)$, and U2AF1 $(n=6)$ using three respective

157 query gene sets of 112, 107, and 96 differentially expressed genes [13]. By comparing SF

158 mutations (MUT) with wild-type (WT) samples, we identified 281, 269, and 285 AS events

159 and 19, 31, and 15 pathways, respectively, for SF3B1, SRSF2, and U2AF1 (Additional File 1:

160 Table S1). Proportions of each AS event type are summarized in Figure 2.A. RI (37.9 $\square 53.7 \%)$

161 was most frequently detected in three cases, and the frequency of A3 events was next

162 (Additional File 2: Table S2). The dominant occurrence of RI and A3 events in our result is 163 consistent with a previous MDS analysis study using rMATS [4,6,13,23-26]. However, in 164 the previous study, a more refined final DAS set from two comparisons using both WT and 165 healthy control samples as controls, were selected [7,23]. When considering a single 166 comparison with WT samples in rMATS as we did, SE showed the largest proportion across 167 the three SF analyses (34.1 59.4\%; Additional File 2: Table S2). When comparing with 168 results from the other two methods (performed in the final section of evaluation), SUPPA2 169 detected A3 (40.3\%) most frequently, followed by SE (28.7\%) and RI (19.4\%) [8]. MISO 170 showed a similar pattern to that of rMATS (SE $25.5 \%$, A3 18.4 \%, and RI 25.7 \%) [9]. Three 171 SFs, SF3B1, SRSF2, and U2AF1, are known to recognize the 3' splice sites (acceptor sites) 172 [15]. Therefore, our method minimized bias toward specific AS event types and reflected the 173 role of spliceosome recognizing cis-elements. SUPPA2 was also able to project the 174 characteristics of the spliceosome. 
175 To delineate pathway regulation for each SF MUT, we presented hallmark pathways highly

176 ranked by stat-P (Figure 2B; further details of stat-P described in the Method section). The

177 heme metabolism (HM) pathway was top-ranked in all three analyses. Coagulation, hypoxia,

178 oxidative phosphorylation, inflammatory response, and estrogen receptor signal pathways

179 were also revealed to be regulated by the three SF MUTs. The previous MDS study used a

180 commercial software, IPA for pathway analysis, which ranked sirtuin signaling as the first

181 and heme biosynthesis as the second [13]. As the sirtuin pathway was absent in the hallmark

182 pathway set, our result is remarkably similar to that of the previous study.

183 For additional validation, we evaluated the discriminative power of our AS event set and

184 compared the enrichment to biological function with the rMATS result. Specifically, we

185 investigated the result of the SF3B1 analysis. As shown in a scatterplot of principal

186 component analysis (PCA), the PSI profile of 281 AS events accurately discriminated

187 between the MUT and WT samples (sensitivity: 100\%, specificity: 96.3\%; Figure 2C). To

188 compare and to evaluate pathway enrichment of our AS event set, we executed rMATS and

189 obtained DAS sets for two conditions, one with the same criteria adopted in the previous

190 MDS study (Cond1; $\mathrm{n}=596)$ and another with a more strict option (Cond2; $\mathrm{n}=367$ ) [13].

191 Previous literature in combination with our findings (Figure 2B) suggests that hematopoietic

192 malignancy, HM, and heme biosynthesis are dysregulated by SF3B1 mutation in MDS or

193 U2AF1 in other blood cancers $[4,6,13,23-26]$. Therefore we selected the HM pathway as a

194 true gene set. ASpediaFI demonstrated the best overall performance from the perspective of

195 both Fisher's exact test $P$-value and Jaccard index in all three SF MUT analyses except

196 SRSF2, where rMATS Cond1 showed the lowest $P$-value (Figure 2D). We additionally

197 examined our AS event genes and their specific functions using the Venn diagram to compare

198 the three sets from ASpediaFI, rMATS Cond2, and HM expansion set (Figure 2E). We chose 
199 rMATS Cond2, which performed better in the SF3B1 analysis over Cond1. We generated the

200 HM expansion set by merging the HM pathway gene set with interacting genes in our PPI

201 network in order to investigate novel candidates for genes regulating the pathway by

202 alternative splicing (more details are described in method). AS event genes of ASpediaFI

203 (Fisher's exact test $P$-value $=0.004$ ) are more significantly enriched in the HM expansion set

204 than in rMATS Cond2 $(P$-value $=0.199)$. Meanwhile, we explored several functional

205 sequence features involved in splicing regions using the ASpedia database. The AS events

206 generated by our analysis were involved in more protein domains, nonsense mediated-decays

207 (NMD), and isoform-specific protein-protein interactions (PPI) than those generated by

208 rMATS Cond1 and Cond2 but contained fewer post-translational modifications (PTM) and

209 repeat regions (Additional File 2: Table S4; Figure 2F) [22].

210 We examined the biological function of spliced genes in two distinct mutually exclusive sets

211 of ASpediaFI $(n=22)$ and rMATS $(n=8)$ overlapping with the HM expansion set

212 (Additional File 2: Table S3). We divided the HM expansion set into two, known genes that

213 belong to the HM pathway and novel genes adjacent to the genes in the HM set. Total events

214 in the exclusive sets were detected at a higher frequency with ASpediaFI. Novel AS genes

215 were also detected more efficiently with ASpediaFI ( $n=17$; rMATS $n=8)$. We identified two

216 known splicing genes NARF and SNCA, that are directly associated with MDS belonging to

217 the HM pathway (Additional File 2: Table S3). Interestingly, only ASpediaFI detected an AS

218 event on the synuclein alpha $(S N C A)$ gene, and the ASpedia database identified the

219 'synuclein' domain in the AS inclusion region (Additional File 2: Table S3), which has been

220 shown to interact with sirtuin 2 [27]. As we already described in previous, sirtuin signaling

221 was not able to detect in our result (Figure 2B). However we successfully identified SE event

222 of the sirtuin signal-associated gene, SNCA and our result implies to engage spliced genes in 
223 both heme metabolism and sirtuin-1 autophagy pathway like previous finding [28]. We also

224 exclusively identified a RI event of NARF in the C-terminal' domain of the 'Iron only

225 hydrogenase large subunit' where the event induces Alu-exon insertion and affects substrate-

226 binding affinity or catalytic activity in MDS [25]. The ASpediaFI results also included more

227 novel AS events (HM expansion set) (47\% of 17) involved in protein domains compared to

228 rMATS (40\% of 5; Additional File 2: Table S3). Among the novel events identified by

229 ASpediaFI, we found a RI event of CDC37. The gene is regulated by Hsp90 during the

230 biogenesis of the active conformation of the heme-regulated eIF2 $\alpha$ kinase, and spliced site is

231 critical to the loss of the 'Hsp90 binding' domain [29]. In summary, ASpediaFI identified

232 more number of novel AS events than rMATS. These findings can be interpreted as evidence

233 that ASpediaFI efficiently detects novel and functionally important AS events.

234 Case study 2: EMT pathway in stomach cancer induced by ESRP1 and the

235 representative AS events.

236 Epithelial regulatory splicing factor, ESRP1, is down-regulated during epithelial-

237 mesenchymal transition (EMT) and plays a critical role in tumor progression [30,31]. We

238 performed analysis on the TCGA STAD RNA-Seq dataset to examine ESRP1-related AS

239 events, associated pathway regulation. Additionally, we investigated the consistency of our

240 results by comparing it with GSEA using gene expression to verify our method by

241 performing integrative analysis. Samples were classified into ESRP1 high $(\mathrm{n}=41)$ and low (n

$242=42)$ groups based on ESRP1 mRNA expression (RPKM). ASpediaFI identified seven

243 pathways and 293 AS events (Additional File 1:Table S1). The PSI profile of the detected AS

244 events provided a powerful discriminatory performance (Sensitivity: 100\%, Specificity: 69\%;

245 Figure 3A). The proportions of five AS event types are presented in Figure 3B. SE was 
246 identified in $66 \%$, and it was three times the sum of (22\%) of A3 and A5. In additional DAS

247 analysis using SUPPA2, SE events (57\%) were detected most frequently. Percentages of five

248 AS types identified by ASpediaFI consistently resembled those detected by SUPPA2, as

249 already uncovered in case study 1. In pathway analysis, ASpediaFI ranked the EMT pathway

250 on top and consequently identified EMT-associated pathways such as 'myogenesis' and

251 'apical junction' (Figure 3C). To compare gene expression-based analysis with ours, we

252 estimated pathway scores for each sample using GSVA from the gene expression profile and

253 compared them with our pathway rankings (Figure 3C) [32]. The GSVA result resembled our

254 rankings except for two pathways, 'IL2-STAT5 signaling' and 'UV response down,' which

255 exhibited lower relevance than EMT and myogenesis.

256 To investigate the biological function and novelty of spliced genes, we compared two AS

257 gene sets inferred from ASpediaFI and SUPPA2 with the EMT expansion set (Figure 3D).

258 The two gene sets were equivalently enriched in the expansion set (Fisher's exact test $P$-value

$259<0.003$ ). When retrieving functional sequences of DAS events from ASpediaFI and SUPPA2

260 (Additional File 2: Table S4), AS events were comparably enriched in protein domains for

261 ASpediaFI (32.5\%) and SUPPA2 (33.1\%). The frequency of NMD was slightly higher in

262 ASpediaFI, and SUPPA2 was better at identifying repeat regions. PTM and PPI were

263 remarkably much more frequently identified by ASpediaFI (37.0\%, 35.8\%) than SUPPA2

264 (29.9\%, 24.0\%). Meanwhile, ASpediaFI exclusively identified more novel AS events $(\mathrm{n}=23)$

265 than SUPPA2 $(n=16)$. Moreover, the novel events identified by ASpediaFI were more

266 involved in protein domains (ASpediaFI: $34.8 \%$ of 23 events, SUPPA2: $25 \%$ of 16;

267 Additional File 2: Table S5). On comparing the DAS sets from ASpediaFI and SUPPA2 with

268 five known EMT or ESRP1-associated splicing signatures [31,33-35], the results of the 
269 Fisher's test and Jaccard indices were notably better for the ASpediaFI DAS set across all

270 signatures (Figure 3E).

271 Notably, our result identified novel events, ENAH SE, FGFR2 MXE, and TCF7L2 SE, which

272 were neither present in the hallmark EMT pathway gene set nor detected by SUPPA2. The

273 three events were also identified in all five splicing signatures (Figure 3E). PSI values of

274 these splicing events exhibit strong correlation coefficients $(|r|=0.62 \sim 0.72)$ with EMT

275 pathway scores calculated by GSVA based on gene expression (Figure 3F). Our three events

276 were also present in the EMT-associated submodule extracted by applying stringent cutoffs

277 (gene $\log 2$ fold change $>2$ and AS $|\mathrm{dPSI}|>0.25$ ) (Figure 3G). Our network revealed the

278 functional interactions of TCF7L2 and FGFR2 with FLNA to be a network hub and to

279 regulate EMT in tumor cells [36]. Occurrence of the representative three AS events in the

280 genomic regions lead to changes in the protein domain, and these were shown to be strongly

281 involved in EMT-associated functions based on previous literature [16,37,38]. ENAH, an

282 actin cytoskeleton regulatory gene, is spliced, and exon11a is skipped on the EVH2 domain

283 (Additional File 3: Figure S1). FGFR2 MXE generates two isoforms: FGFR2-IIIb, which is

284 exclusive to epithelial cells and FGFR2-IIIc, which causes a switch from the mesenchymal

285 isoform and induces a change in ligand binding specificity, thereby regulating cell

286 proliferation and differentiation (Additional File 3: Figure S1) [16]. TCF7L2 SE is present in

287 the 'N-terminal CTNNB1 binding' region, FGFR MXE in the 'Immunoglobulin I-set domain,'

288 and ENAH in the 'EVH2 domain' (Additional File 3: Figure S1). TCF7L2 SE in the

289 CTNNB1 binding domain has an impact on the activity of Wnt/ $\beta$-catenin target genes, and its

290 deficiency was verified as the depletion of a proliferative cell compartment in the intestinal

291 epithelium in mouse [38]. Its switch-like exon usage was revealed to be associated with

292 invasive and mesenchymal-like breast tumors [37]. 


\section{Case 3: Splicing events uncover neuronal development by RBFOX1 knockdown.}

294 AS events mediated by RNA-binding protein RBFOX1 regulate neuronal development and

295 pertain to brain diseases like autism [5,14]. We analyzed the RBFOX1 knockdown RNA-Seq

296 dataset of primary human neural progenitor cells, which included five RBFOX1 knockdown

297 samples and five control samples. In order to be consistent with the previous study, we

298 changed the reference pathway gene set to GO level 5 [5]. Finally, ASpediaFI identified 291

299 AS events and nine pathways (Additional File 1: Table S1). A3, RI, and SE were frequently

300 detected, and MXE was the least predominant (Figure 4A). To verify the result, our AS genes

301 were compared with three relevant gene signatures (autism, RBFOX1, and RBFOX2) and

302 three controls (mitrochondrial, ataxia, and epilepsy) obtained from the previous study using

303 the Jaccard index (Figure 4B) [5]. Relevant signatures were collected from spliced gene

304 analysis results of autism $(n=247)$, RBFOX1 $(n=1103)$, and RBFOX2 $(n=1681)$. Controls

305 were randomly selected from known gene sets, mitochondrial $(n=310)$, ataxia $(n=51)$, and

306 epilepsy (n=46). Relevant signatures exhibited higher similarity to our AS gene set in terms

307 of the Jaccard index compared to that of the control set (Figure 4B). In accordance to the

308 pathway ranking of our analysis, neurogenesis, neuron differentiation, and nervous system

309 development pathways were induced in response to RBFOX1 knockdown (Additional File 1:

310 Table S1).

311 To evaluate the pathway detection performance, we compared our results with those of the

312 previous study [5]. The study generated two sets of SE events with differential exon inclusion

313 and exclusion. The biological process was also investigated by GSEA for each AS set. In

314 further GSEA using the AS event set, the previous study identified a subnetwork regulated at

315 the gene expression level. We combined the two AS sets into one 'DAS' set and used a co- 
316 expressed subnetwork gene set named 'Blue module' from the previous study [5]. To verify

317 the gene set enrichment in biological process detection potential, these two gene sets were

318 compared with the highly scored genes (permutation $P$-value $<0.05$ ) identified by our

319 method. We chose the top five GO terms from the GSEA result of the three gene sets and

320 computed their percentile ranks (Figure 4C). The 'Blue module' was enriched in cell

321 migration and motility but failed to detect neuronal differentiation and neurogenesis. In

322 contrast, we observed that nervous system development was more enriched than cell

323 migration and motility in the 'DAS' gene set (Figure 4C). Unlike these two signatures,

324 ASpediaFI successfully identified the most relevant biological processes associated with

325 neuronal development on top percentile rank GO terms except for post-transcriptional

326 regulation, viral life cycle, and mitotic cell cycle (Figure 4C, the first column FI). This result

327 illustrates the advantage of our integrative approach based on both gene expression and PSI

328 profiles and the limitation of independent gene set tests (Blue module and DAS) for

329 analyzing splicing-associated biological functions.

330 We identified an RBFOX1-associated module within the heterogeneous network (Figure 4D).

331 The subnetwork included AS events of ROBO1 and CLIP1, both of which had neural-

332 regulated micro-exons (exons with $3 \square 27 \mathrm{nt}$ ) involved in an AS interaction network

333 associated with the autism spectrum disorder in the previous study [14]. Among our AS

334 events, three micro-exon events (AP2M1, CLASP1, ROBO1) were detected as neural-

335 regulated in the previous study. In particular, ROBO1 exon 18 skipping is known to induce

336 helical domain exclusion and is involved in the loss-of-function of the ROBO1-SLIT2

337 signaling, thereby modulating neurogenesis and proliferation (Figure 4E). In our result, exon

338 exclusion of ROBO1 was significant (permutation $P$-value $=0.001, \mathrm{dPSI}=-0.265$;

339 Additional File 1: Table 1) and moderately correlated with the GSVA scores of the 
340 REACTOME ROBO receptor signaling pathway $(r=-0.53)$. Meanwhile, exonic regions in

341 our AS sets were involved in the protein domain (45.7\%) and isoform-specific interactions

342 (21.3\%) (Additional File 2: Table S4). SE events by RBFOX1 knockdown induce an

343 increase in the alteration of the protein domain, NMD, and repeat region, but decrease PTM

344 and PPI.

345 Performance comparison using SF3B1-associated MDS RNA-Seq dataset

346 The ability of the four different methods to detect DAS was evaluated by using the case study

3471 database (details described in Methods). We selected an additional three programs, rMATS,

348 MISO, and SUPPA2 for comparison [7-9]. We obtained four DAS sets from ASpediaFI (281

349 events at 194 genes), rMATS (596 events at 415 genes), MISO (685 events at 461 genes),

350 and SUPPA2 (129 events and 99 genes) that were extracted from the results. To evaluate the

351 functional enrichment of the detected DAS genes, we assessed the enrichment in the HM and

352 expansion gene set, which are clinically known pathways regulated in MDS SF3B1 MUT

353 samples $[4,13,23-26]$. The ASpediaFI result showed the best performance based on metrics

354 like Fisher's exact test $P$-value and $F_{1}$ score (Figure 5A, Additional File 3: Figure S2).

355 Between the two gene sets (Figure 5A), the ASpediaFI recall (0.175) in the HM expansion set

356 was much better than that in HM (0.04). This result suggests that our method provides better

357 performance for identifying AS events in a novel gene set like HM expansion compared to

358 the other three tools (Figure 5A, Additional File 3: Figure S2). Meanwhile, to reduce the bias

359 of comparing DAS sets with a different number of events, we modified the criteria for

360 differential splicing such that the top 300 ranked AS events after filtering out events with |

$361 \mathrm{dPSI} \mid<0.1$ are selected. This strategy substantially decreased the total counts in MISO and

362 rMATS. ASpediaFI exhibited the best performance across Fisher's exact test, precision, 
363 recall, and $F_{1}$ than others (Additional File 2: Table S6). Regardless of the numbers of DAS

364 events based on relaxed or strict thresholds, ASpediaFI consistently outperformed the other

365 methods in detecting biologically relevant DAS events enriched in HM and expansion set.

367 Performance evaluation on simulated datasets

368 To evaluate the ability of ASpediaFI to detect biologically relevant DAS events under a

369 simulated environment, we generated a simulation dataset imitating the genomic

370 characteristics of the MDS MUT and WT datasets. To artificially induce DAS events, we

371 used the intersection DAS set identified by MISO (892 genes), rMATS (640 genes), and

372 SUPPA2 (623 genes) as the ground truth for the evaluation (Figure 5B). Transcript counts of

37320 replicates per condition were simulated from the distributions estimated by SF3B1 MUT

374 and WT samples. We assigned the pre-determined relative isoform abundances for 125

375 ground truth AS genes collected from the intersection of three results, while those of other

376 genes were drawn from the uniform distribution. ASpediaFI was excluded from generating

377 these simulated RNA-Seq data for the blind test.

378 The ability of the four methods to detect previously defined ground truth AS events was

379 verified. To measure the discriminative power, we computed AUC, AUC-ROC, and AUC-PR.

380 As ASpediaFI runs DRaWR generating stationary probabilities for two stages, we used both

381 stat-P's for the comparison. The first stage (S1) stat-P values were computed for the whole

382 AS events, and the final stat-P values (S2) were considered as refined ranks, enhancing the

383 internal performance. ASpediaFI S1 achieved a higher AUC-ROC value of 0.79 than MISO

384 (0.64), rMATS (0.67), and SUPPA2 (0.67) (Figure 5C). The performance difference

385 manifested the overall false-positive rate $(0 \square 0.75)$. Not surprisingly, ASpediaFI S2 was 
386 better $(\mathrm{AUC}-\mathrm{ROC}=0.94)$ than $\mathrm{S} 1$. Moreover, we assessed the accuracy based on the lower

387 number of samples per condition between the four methods. Compared to the fully simulated

388 dataset (20 replicates per condition), the three other methods showed consistent performance

389 for the smaller sample sizes (10 replicates) (Additional File 3: Figure S3). In the smallest

390 dataset $(n=5)$, our AUC-ROC decreased to 0.73 from 0.78 , but the difference of true-positive

391 rate was still maintained over the most important region (false-positive rate $0 \sim 0.25$ ).

392 Although the AUC values of ASpediaFI slightly decreased, followed by sample size, S1

393 consistently exhibited superior performance compared to the other methods across the three

394 simulated datasets of different sizes (Figure 5D, Additional File 3: S3). Additionally, the

395 discriminative power of S2 remained reasonably stable under varying sample sizes.

396 We further examined the biological relevance of DAS events detected from the fully

397 simulated dataset. As the simulation RNA-Seq samples were derived from SF3B1 MUT and

398 WT samples, and as the ground truth AS events were defined based on the MDS sample

399 analysis, we expected that the simulated samples would maintain the characteristics

400 associated with the HM pathway dysregulation. Before DAS identification, we validated our

401 assumption using GSEA with the gene expression profile. Finally, the HM pathway was

402 consistently observed as the most significantly enriched pathway in the simulated dataset, as

403 in case study 1 (adjusted $P$-value $=0.06$; Figure 5E). The previously identified hypoxia and

404 MTORC1 signaling pathways were also retained (adjusted $P$-value $=0.15,0.17)$. Next, to

405 make a fair comparison with ASpediaFI (DAS events $n=499$ ), we identified the top 500

406 DAS after filtering $\mid$ dPSI $\mid>0.1$ using the other three methods. ASpediaFI exhibited a higher

407 degree of enrichment in both the HM and expansion sets compared to the other three methods

408 based on Fisher's exact test $P$-value and $F_{1}$ score (Figure 5F, Additional File 3: Figure S4).

409 When stricter statistical cutoffs (FDR $<5 \%$ for rMATS and SUPPA2, Bayes Factor $\geq 5$ for 
410 MISO) were applied to the other three methods, $210 \square 280$ AS events were identified

411 (Additional File 2: Table S7). rMATS showed the highest enrichment according to Fisher's 412 exact test $($ HM $P$-value $=0.015$, expansion $P$-value $=0.00085)$. Nevertheless, we observed

413 that ASpediaFI showed better performance with respect to the $F_{1}$ score (ASpediaFI 0.2,

414 rMATS 0.107) of HM expansion than rMATS. It implies that our method detected novel AS

415 events that are not present in the curated gene set. Overall, based on our benchmarking

416 analyses using computationally simulated datasets, ASpediaFI showed a higher potential for

417 identifying biologically-relevant DAS events.

\section{DISCUSSION}

420 After the advent of next-generation sequencing, various novel methods for DAS analysis

421 have been developed. Although approaches for DAS event identification have improved in

422 accuracy, it is still a challenge to interpret the biological relevance as well as integration with

423 regulatory mechanisms with DAS events. Here, we suggest an integrative method, ASpediaFI,

424 to systematically identify AS events, co-expressed genes, and pathways regulated by the

425 transcriptome. ASpediaFI ranks AS events, pathways, and genes, and also intuitively

426 provides functional interactions in the form of an interaction network. It enables the users to

427 understand global regulation and specific pathways by spliceosome and to choose more

428 relevant AS events as markers.

429 In order to verify the intrinsic ability of ASpediaFI, we analyzed three case study datasets of

430 MDS, STAD, and RBFOX1 knockdown. Pathway analysis results using our method

431 presented remarkable consistency with GSEA or GSVA using the gene expression profile.

432 This consistency can be attributed to the fact that our analysis starts with getting a query from 
433 the DEG set and performs RWR via a heterogeneous network that includes correlated AS

434 with gene expression. Despite tumor heterogeneity in case 1, the high number of replication

435 (total samples $\mathrm{n}=84$ ) facilitated the identification of AS events, their interacting genes, and

436 pathway-level regulation by SF MUT. Next, we succeeded in identifying the gastric cancer

437 EMT subtype based on the DAS. The subtype was revealed to be the one with the poorest

438 survival among the four known gastric cancer subtypes [39]. Even though we identified a

439 small size DAS set of around 200 events, our result demonstrated the discriminative power to

440 classify samples by SF regulation (Figure 2C, Figure 3A). In particular, the three

441 representative AS events, ENAH, FGFR2, and TCF7L2, that were identified only by

442 ASpediaFI, had the potential to effectively classify the gastric cancer EMT subtype. It was

443 comparable to the previous classification of the EMT subtype using the gene signature of

444 over 300 genes [39]. In case 3, the previous RBFOX1 study performed GSEA and network-

445 based module identification for each DEG and DAS sets [5]. This previous approach required

446 the identification of relatively large DAS sets $(n=996)$. To uncover relevant biological

447 process, the large size DAS set was divided into subsets by SE type or exon inclusion, and

448 multiple sets were respectively used for GSEA. Moreover, independent analyses of DEG and

449 DAS could not be interlinked to explain the systematic interactions between AS events,

450 although the previous study successfully revealed the regulation of neuronal development by

451 RBFOX1. Moreover, the pathway revealed using the two gene sets used in the previous study

452 was complementary for uncovering neuronal development by RBFOX1, as already shown

453 (Figure 4C). The multiple independent tests and complementary result highlight the

454 advantage of our method.

455 In the case studies, our method correctly identified the AS type usage based on the role of SF

456 with respect to recognizing donor and acceptor sites. In the previous study comparing several 
457 DAS methods, exon-based approaches mostly showed the best AU-ROC in terms of the SE

458 event among the four AS types compared to the isoform-based methods [12]. Moreover, SE

459 is the predominant type in the human gene model, and its PSI value calculated from three

460 junctions and exons is more stable than A3 and A5 calculated from transcript regions

461 narrower than SE. Therefore, exon-based DAS analysis applications have the potential to

462 include bias according to AS type than isoform-based methods [12]. U2AF1, like SF3B1, is a

463 member of the U2 complex and is known to recognize the $3^{\prime}$ dinucleotide motif AG, so A3

464 and RI could increase in the background of U2 complex member deficiency [6]. In case study

4651 , our result mirrors the characteristics of spliceosomes. Among the four tools we used, the

466 AS type proportions of SUPPA2 resembled ours in case studies 1 and 2. The results of case

467 study 2 and 3 were similar to previous results that identified the induction of SE events by

468 ESRP1 and RBFOX1 [5,30,31]. In contrast to our result, rMATS most frequently detected SE

469 events in the case studies. We deduced that the previous study on the MDS dataset had to

470 carry out two comparisons with two different controls to avoid the SE bias [13]. When

471 calculating the ratios of SE over the sum of A3 and A5 from several EMT-associated DAS

472 results, rMATS (SE $n=239$, FDR $<10 \% ; 18.8$ times) and MADS+ (20 times) detected SE

473 events at a higher frequency than previous analyses using Affymetrix exon 1.10 microarray

474 (8.8 times) and RNA-Seq dataset considering sequence motif (3.6 times) and ours (3.1 times)

$475[7,30,31,34]$. That is, ASpediaFI provided results with a minimal bias toward SE, similar to 476 SUPPA2.

477 To evaluate the performance of ASpediaFI and to compare it with other tools, we selected

478 three analysis tools. In the early stage, we tried to add JUM, but we decided not to use it due

479 to the extremely lower number of DAS passing the FDR threshold $(<5 \%)$. JUM can identify

480 novel structured AS events not present in the transcriptome annotation [10]. We speculated 
481 that the advantage of JUM with respect to identifying novel events paradoxically reduced the

482 detection of proper DAS events. Meanwhile, we chose the HM pathway as a gold standard

483 for the performance evaluation of the analysis of the MDS dataset, based on the evidence

484 from case study 1 and multiple previous clinical MDS studies [4,13,23-26]. The previous

485 studies consistently reported the deficiency in heme biosynthesis and iron homeostasis due to

486 splicing upon analyzing $12 \square 100$ samples. Unfortunately, the previous four splicing

487 signatures identified from SF3B1 MUT samples did not have uniform quality, and identified

488 AS signatures were small size $(n=20 \square 202)$ except for one $(n=1403)[4,13,24,40]$. However,

489 we tried to perform Fisher's exact test and Jaccard index for these four splicing signatures

490 with our ASpediaFI AS results, Iron homeostasis transport, inflammatory response, HM and

491 expansion set to evaluate the functional relevance based on previous studies $[4,6,13,23-26]$.

492 ASpediaFI showed remarkable consistency (Fisher $P$-value $<0.0003$ ) with three signatures

493 except for the smallest sized signature $(\mathrm{n}=20 ; P$-value $=1) . \mathrm{Next}$, the HM and expansion set

494 represented the best enrichment $(\mathrm{HM}$ Fisher median $P$-value $=0.1$; expansion $P$-value $=0.1)$

495 than others (Iron homeostasis transport $P$-value $=0.3$; Inflammatory response $P$-value $=0.8$ ).

496 Based on these results and previous studies, we concluded that DAS events induced by

497 SF3B1 MUT in MDS are enriched in the HM pathway and continued our evaluations.

498 During the evaluation using a simulated dataset, our method consistently showed the best

499 performance compared to the other three tools. We tried to generate simulated RNA-Seq

500 samples imitating actual MDS characteristics. We evaluated our capability to detect

501 biologically relevant AS events in a dedicated setup, including the estimation of MUT and

502 WT transcript count distributions and recurrent detection of DAS. Finally, we worked on

503 benchmark evaluation as well as investigation of HM pathway enrichment. ASpediaFI

504 generated the best ROC curves and presented a true-positive rate difference continuously 
505 across a long-range of false-positive rates $(<0.75)$ (Figure 5C). In the datasets with smaller

506 sample sizes ( $\mathrm{n}=10$ and 5), our method still showed the best result. For the evaluation of our

507 tool, we used both S1 and S2 scores (Figure 5C). However, the second stage RWR is

508 performed to rescore only AS events selected in S1. Therefore, S1, which is run on total AS

509 events, is more suitable for comparison, and the outstanding achievement of S2 should be

510 carefully interpreted. To achieve the best performance for each tool, we optimized parameters,

511 such as FDR, dPSI, or BF and generally used cutoff values of other studies [7,10,24].

512 Sometimes, we removed additional filtering (dPSI) and only considered numerical scores

513 (FDR or BF) from each tool. Despite these attempts, MISO demonstrated a weak

514 performance in AUC-PR and HM enrichment. While rMATS showed the best performance in

515 HM enrichment, ASpediaFI presented the best overall performance.

516 Our novel integrative approach using both PSI and gene expression offers a unique advantage.

517 Instead of independent multiple GSEA tests for DAS and DEG, ASpediaFI systemically

518 elucidates interactions between AS and genes and delineates pathway regulation. Another

519 novel characteristic is its ability to identify relevant pathways using small size DAS sets. In

520 contrast, other studies analyzed approximately $500 \square 1000$ AS events to reveal biological

521 functions, and investigated pathways by dividing sets into inclusion and exclusion events.

522 However, our method required fewer than 300 AS events to identify specific pathways in the

523 three case studies. The total counts of our AS results are close to the recommended gene set

524 size of at least 15 to at the most 200 genes [18] essential to identify splicing markers. Besides,

525 there are additional advantages. AS event IDs of ASpediaFI results could be used to query the

526 ASpedia database to explore comprehensive functional sequence features like protein domain,

527 NMD, and isoform-specific interaction. Our tool has no dependency on any organisms or

528 alignment tool. ASpediaFI refers dataset or file formats-BAM file, gene model, PPI, or gene 
529 sets-widely used in gene expression analyses. Moreover, our method supports fast

530 execution time. The most time-consuming jobs to read bam files are provided with multi-

531 thread option, and the principal analysis of DRaWR S1 and S2 except preprocessing is

532 executable in a PC environment (RAM 16GB, CPU $3.40 \mathrm{GHz}$ and 2 minutes of execution

533 time for case 1 SF3B1 dataset with total 82 RNA-Seq samples).

534 There are several limitations to ASpediaFI. Our method requires a reference interaction

535 network and gene set. Prior to network establishment, our method involves filtering based on

536 several criteria, including low gene expression and standard deviation of PSI. While it is

537 effective at excluding unreliable PSI values calculated from lowly expressed genes, it is

538 subject to the loss of lowly expressed true-positive AS events. As shown in the performance

539 evaluation, our application needs at least five samples per condition to obtain a stable result.

540 Additionally, ASpediaFI requires at least three samples per condition to calculate the

541 correlation coefficient between the AS and gene. In a further development, we expect to

542 improve the applicability of our method to a small dataset with less than five replicates or

543 even without replication. Moreover, we also hope to extend our algorithm to the analysis of

544 novel conditions like time-series or continuous statement of SF.

\section{CONCLUSION}

547 In this study, we developed ASpediaFI and analyzed RNA-Seq datasets to verify the

548 capability of our method to interpret biological processes regulated by splicing. As shown in

549 the three case studies, ASpediaFI successfully identified AS events and relevant pathways

550 involved in query DEGs. On comparison with three other three programs, ASpediaFI showed 
551 superior performance, as determined by the AUC-ROC and AUC-PR. We expect that

552 ASpediaFI will uncover novel roles and global regulation of SFs.

554 MATERIAL AND METHODS

\section{Data preparation}

556 ASpediaFI requires input files, including a gene model, RNA-Seq BAM files, gene

557 expression profiles, pathway gene sets, and a global gene-gene interaction network. First, AS

558 events were identified using a gene model of a GTF file and classified into the following five

559 types: alternative $5^{\prime}$ splice site (A5), alternative $3^{\prime}$ splice site (A3), skipping exon (SE),

560 mutually exclusive exons (MXE), and retained intron (RI). PSI values of the identified events

561 were calculated based on read counts mapped to exons and splice junctions. ASpediaFI uses

562 these AS events, pathway gene sets, and a gene interaction network as reliable sources of

563 interactions for the construction of a heterogeneous network. Our heterogeneous gene

564 interaction network refers to a reference gene interaction. In our analysis, we collected and

565 curated reference-based interaction databases (BIND, DIP, HPRD, and REACTOME) to

566 build a reference human gene interaction compendium, which contains 10,647 genes and

56754,037 interactions $[19,41-43]$. We also referred to public pathway databases (hallmark,

568 REACTOME, and KEGG) and obtained a total of 910 human pathway gene sets $[18,19,44]$.

\section{Heterogeneous network construction}

570 Based on the biological information inferred from the RNA-Seq datasets and public databases,

571 ASpediaFI constructed a heterogeneous network composed of gene nodes and two types of

572 feature nodes: AS event and pathway. The heterogeneous network allows interactions 
573 between genes and between gene and feature node of gene-AS and gene-pathway. ASpediaFI

574 refers to a reference network to connect gene interactions. Gene-gene interaction edges were

575 weighted with the absolute value of the Pearson correlation coefficient calculated from gene

576 expression. Gene-AS interaction edges are connected if the absolute value of the Spearman

577 correlation coefficient between gene expression and PSI exceeds a user-defined threshold.

578 Due to the nonlinear relationship between gene expression and PSI values, we used the

579 Spearman correlation coefficient as a measure of association strength for gene-AS [45].

580 Finally, gene-pathway edges are weighted to 1 if the corresponding gene belongs to the

581 corresponding pathway gene set.

\section{Query-specific subnetwork identification using DRaWR}

583 To explore the important submodules, we employed DRaWR, which is the extension of

584 random walk with restart (RWR) using a heterogeneous network consisting of feature nodes

585 [20]. The DRaWR algorithm performs two-stage RWR in which a functional subnetwork is

586 extracted in the first stage, and nodes in the subnetwork are ranked by associations with a

587 query gene set in the second stage (Figure 1B).

588 Let $A$ be an adjacency matrix representing our heterogeneous network. The adjacency matrix

589 can be expressed as:

$$
M=\left[\begin{array}{lll}
M_{g g} & M_{g a} & M_{g p} \\
M_{a g} & M_{a a} & M_{a p} \\
M_{p g} & M_{p a} & M_{p p}
\end{array}\right]
$$

590 where submatrices $M_{g g}, M_{g a}$, and $M_{g p}$ exhibit edges between gene-gene, gene-AS, and gene-

591 pathway. Therefore, the entries of $M$ can be written as: 


$$
\begin{aligned}
& m_{g_{i} g_{j}}=\left\{\begin{array}{r}
\left|r_{P}\left(g_{i}, g_{j}\right)\right|, \text { if found in the gene interaction network } \\
0, \text { otherwise }
\end{array}\right. \\
& m_{g_{i} a_{i}}=\left\{\begin{array}{r}
\left|r_{s}\left(g_{i}, a_{i}\right)\right|,\left|r_{s}\left(g_{i}, a_{i}\right)\right|>\tau \\
0, \text { otherwise }
\end{array}\right. \\
& m_{g_{i} p_{i}}=\left\{\begin{array}{l}
1, \text { if a gene is in a pathway gene set } \\
0, \text { otherwise }
\end{array}\right.
\end{aligned}
$$

592 where $r_{P}$ and $r_{S}$ are the Pearson and Spearman correlation coefficients, respectively and $\tau$ is a 593 user-defined threshold. Note that $m_{a_{i} a_{j}}, m_{a_{i} p_{i}}$, and $m_{p_{i} p_{j}}$ are all zero as there are no edges

594 among feature nodes. Before running RWR, each nonzero submatrix is normalized such that 595 its entries total 1 , and the whole normalized adjacency matrix is again normalized by column 596 to obtain a transition matrix $T$.

597 Given a transition matrix, the RWR algorithm can be formulated as:

$$
\boldsymbol{\pi}^{t+1}=(1-c) T \pi^{t}+c v
$$

598 where $\pi_{i}^{t}$ is the probability that the walker will stay at node $i$ after the $t$ th iteration, $c$ is the 599 probability of restart, and $v_{j}$ is the probability of restarting at a node $j$. That is, for a query 600 gene set $Q, v_{j}$ is $\frac{1}{|Q|}$ if $j \in Q$ and 0 otherwise. We assumed $\boldsymbol{\pi}^{\mathbf{0}}$ to be a uniform probability 601 vector such that $\pi_{i}^{0}=\frac{1}{n}$, where $n$ is the number of all nodes in a heterogeneous network.

602 In the first stage of DRaWR, RWR is run twice, once (Stage 1; S1) with a query gene set and 603 another (Stage 2; S2) with all genes in the heterogeneous network as the restart set. The 604 difference between the stationary probabilities (stat-P) in the two runs, say $\widehat{\pi_{Q}}-\widehat{\pi_{B}}$, is a 605 measure of relevance to a query gene set and used to rank AS event nodes and pathway nodes 606 altogether. 
607 Prior to stage 2, ASpediaFI extracts a query-specific subnetwork composed of gene nodes

608 and the user-defined number of highly ranked AS event and pathway nodes. The adjacency

609 matrix of the subnetwork can be expressed as:

$$
M^{\prime}=\left[\begin{array}{lll}
M_{g g} & M_{g a^{\prime}} & M_{g p^{\prime}} \\
M_{a \prime g} & M_{a \prime} a^{\prime} & M_{a p^{\prime}} \\
M_{p^{\prime} g} & M_{p^{\prime} a^{\prime}} & M_{p^{\prime} p^{\prime}}
\end{array}\right]
$$

610 where $a^{\prime}$ and $p^{\prime}$ denote AS event and pathway nodes retained in the subnetwork. The second-

611 stage RWR is performed on the subnetwork in the same way as stage 1 to calculate stat-P and

612 produce final rankings of genes, pathways, and AS events.

\section{Evaluation of two-stage DRaWR and permutation test}

614 ASpediaFI carries out a $k$-fold cross-validation at each stage of RWR to evaluate the

615 performance of the DRaWR algorithm, in the same way as mentioned in the previous study

616 [20]. A query gene set is partitioned into the user-defined number of subsets. For each subset,

617 RWR is run with the remaining genes as the restart set to compute AUC (area under the curve)

618 using the subset as true class labels and stat-P's as predictions. In our analysis, we compared

619 the average AUC at two stages for tuning parameters.

620 While the DRaWR algorithm removes feature nodes having low stat-P values under cutoff

621 derived from querying a gene set before the second stage, all gene nodes are retained in the

622 initial network and only provide their final relevance scores. In order to reduce the

623 background effect of scoring and to filter out false positives, we included the permutation test

624 on gene nodes in the evaluation procedure [46]. ASpediaFI runs $N$ iterations of the second-

625 stage random walks, in each of which a randomly sampled gene set of the same size as a

626 query gene set is used as the restart set. The permutation $P$-value of gene node $i$ is 


$$
P_{i}^{\text {perm }}=\frac{1}{N} \sum_{n=1}^{N} I\left(\hat{\theta}_{i}^{n}>\hat{\pi}_{i}\right)
$$

627 where $\hat{\theta}_{i}^{n}$ is the second-stage stat-P of node $i$ when a randomly sampled gene set is given as a

628 query, and $I$ is an indicator function which gives 1 if $\hat{\theta}_{i}^{n}>\hat{\pi}_{i}$ and 0 otherwise. ASpediaFI

629 refers to stat-Ps as a score for ranking and selecting feature nodes, and permutation $P$-values

630 for choosing pathway-related genes.

631

632 RNA-Seq dataset preparation for case studies

633 Case study 1: The first case study was an RNA-Seq dataset (GEO accession number:

634 GSE114922) from bone marrow-derived CD34+ hematopoietic progenitor cells of 84

635 patients with myelodysplastic syndrome (MDS) [13]. Patients exhibited hotspot mutations in

636 three SF SF3B1 $(n=28)$, SRSF2 $(n=6)$, and U2AF1 $(n=8)$. We first assessed the quality of

637 reads using FastQC v0.11.5, and aligned to the GRCh38 genome and the reference gene

638 model GENCODE v31 using STAR v2.6.1b to follow the GDC pipeline with customized

639 options: outFilterType $=$ BySJout, alignEndsType $=$ EndToEnd,

640 alignSoftClipAtReferenceEnds $=$ No, alignIntronMax $=10000$, alignMatesGapMax $=10000)$

641 [47]. Gene expression profile was evaluated by RSEM v1.3.0 [48]. We calculated the PSI

642 (percent spliced-in) profile from BAM files based on AS events derived from the input gene

643 model. To extract the query gene set, differential expression analysis between the mutated

644 and wild-type samples was performed using limma v3.42.0 [49]. The ASpediaFI analysis was

645 run with the following options:

646 - restart (restart probability): 0.7 
- num.folds (number of folds for cross-validation): 5

648 - num.feats (number of features to be retained in a subnetwork): 300

649 - low.expr (threshold average FPKM of genes): 1

650 - low.var (threshold variance of AS events): NULL

651 prop.na (threshold proportion of missing PSI values): 0.05

652 - prop.extreme (threshold proportion of extreme PSI values -0 or 1): 1

653 - cor.threshold (threshold Spearman's correlation coefficient between genes and AS

$654 \quad$ events): 0.4 .

655 Based on this, we reconstructed three AS-gene interaction subnetworks regulated by three SF

656 mutations from the second stage result of DRaWR. Additionally, highly-scored genes

657 (permutation $P$-values $<0.05$ ) were selected along with neighboring AS event nodes.

658 We further investigated the characteristics and biological relevance of the identified AS

659 events. First, we classified the MDS samples into two groups, SF WT and MUT using the

660 PSI profiles of identified DAS events. We performed hierarchical clustering with complete

661 linkage on the Euclidean distance matrix of the PSI profiles to evaluate the discriminative

662 performance, confirmed by principal component analysis (PCA). Next, we used rMATS to

663 detect DAS between SF3B1 MUT and WT and compared it with our result. Based on

664 previous MDS study analysis condition, we setup rMATS cutoffs (Cond1: $\mid$ dPSI $\mid>0.1 \&$

665 FDR < 0.05) [13]. The number of DAS identified by rMATS Cond1 is over twice of our AS

666 result. To make similar condition, we additionally performed rMATS of more stringent cutoff

667 conditions. We first applied the same thresholds (Cond1: $\mid$ dPSI $\mid>0.1 \&$ FDR $<0.05)$ to

668 follow the methodology used in the previous study. The second thresholds (Cond2: $|\mathrm{dPSI}|>$

$6690.1 \&$ FDR $<0.0001)$ were determined so that the number of DAS events was similar to the 
670 ASpediaFI result. As our method refers to PPI genes to identify all interactions, only AS

671 events in genes in our PPI compendium were considered to reduce the bias introduced using

672 different background genes. We used the Fisher's exact test and Jaccard index to measure

673 how the results of ASpediaFI, rMATS Cond1, and Cond2 are enriched in the heme

674 metabolism (HM) pathway, which was highly ranked in the previous study. Additionally, we

675 defined a novel HM gene set 'HM expansion set' to test whether AS events interact with

676 genes in the HM pathway and participate in the corresponding biological process. The HM

677 expansion set included both HM genes and their neighbor genes derived from our gene

678 interaction network. Fisher's exact test and Jaccard index were also computed for the HM

679 expansion set. To investigate the functional importance of AS genomic regions, we

680 interrogated protein domain, NMD, and other sequential features of AS events using the

681 ASpedia database for ASpediaFI, rMATS, Cond1, and Cond2 [22].

682 Case study 2: We chose the TCGA stomach adenocarcinoma (STAD) level 3 RNA-Seq

683 dataset as another real dataset to investigate AS events and biological processes associated

684 with ESRP1, a key splicing factor that regulates epithelial-mesenchymal transition (EMT)

685 across multiple cancer types [2,7,50]. Of the 415 STAD patients, the highest and lowest $10 \%$

686 mRNA expression samples of ESRP1 were classified as ESRP1-high and ESRP1-low groups,

687 respectively. Due to the absence of BAM files, we used SUPPA2 v2.3, as was done in the

688 previous study, and we also used a gene model referred UCSC known genes to generate PSI

689 profiles [51]. Statistical test for differential expression between the two groups was

690 performed using limma to obtain a query gene set. We conducted the ASpediaFI analysis

691 with the following options: restart $=0.7$, num.folds $=5$, num.feats $=300$, low.expr $=1$,

692 low.var $=$ NULL, prop. na $=0.05$, prop. extreme $=1$ and cor $\cdot$ threshold $=0.5$. To compare our

693 result, we performed DAS analysis using SUPPA2 diffSplice with the following options: 
694 nan-threshold $=10$, area $=1000$ and lower-bound $=0.05$ [8]. SUPPA2 DAS set was obtained

695 by selecting AS events with $\mid$ dPSI $\mid>0.1$ and adjusted $P$-value $<0.1$. Next, we extracted an

696 EMT-associated subnetwork from the final stage produced by DRaWR. To decrease the

697 network size, we filtered out gene nodes with permutation $P$-values not less than 0.05 .

698 Similarly, we tested the discriminative power of our DAS events by classifying STAD

699 samples based on the Euclidean distance matrix of their PSI profiles using hierarchical

700 clustering with average linkage. Meanwhile, we test how much our pathway result identified

701 by ASpediaFI is consistent with GSEA analysis using gene expression profile. Our pathway

702 result was collected by rankings determined by ASpediaFI. For analysis result using gene

703 expression, we calculated sample-level pathway activity scores executing gene set variation

704 analysis (GSVA) [32]. Difference of GSVA scores between high and low groups was tested

705 by Wilcoxon rank-sum test. Next, we compared ASpediaFI with other DAS test method. The

706 results from the two applications, ASpediaFI and SUPPA2, were compared using Venn

707 diagram, Fishers' exact test, and Jaccard index calculated from five EMT or ESRP1-

708 associated splicing gene signatures [31,33-35]. Like in case study 1, AS event sets for two

709 conditions were chosen to overlap with global PPI genes. As in the first case study, we

710 compared the sequential features of AS events detected by ASpediaFI and SUPPA2 by

711 retrieving from the ASpedia database.

712 Case study 3: The last RNA-Seq data (GEO accession number: GSE36710) comprised five

713 replicates of the shRBFOX1 (RBFOX1 knockdown) and shGFP (control) cell lines [5].

714 Single-end RNA-Seq reads aligned to the GRCh37 genome and the reference gene model

715 Ensembl v71 using STAR v2.6.1b with the same options as in case study 1. We calculated

716 gene expression and PSI values using RSEM and our quantification tool. A query gene set 
717 was obtained from the DEG test between RBFOX1 knockdown and control groups using

718 limma. The following options were selected for the ASpediaFI workflow: restart $=0.7$,

719 num.folds $=5$, num.feats $=300$, low.expr $=1$, low.var $=$ NULL, prop.na $=0.05$, prop.extreme

$720=1$, cor.threshold $=0.8$. We then interrogated an RBFOX1-regulated subnetwork. From the

721 final network produced by the DRaWR algorithm, we retained gene nodes with permutation

$722 P$-values less than 0.05 and their neighboring AS event nodes.

723 To examine the enrichment of our AS genes in known neuronal genes, we calculated the

724 Jaccard index between our AS gene set and known gene signatures, as done in the previous

725 study [5]. We prepared three published gene signatures containing genes inferred from

726 transcriptomic analysis of RBFOX1 and RBFOX2, and those showing RBFOX1-dependent

727 splicing in autism spectrum disorder (ASD) brains [52-54]. We also compared with three

728 control gene signatures - mitochondria, epilepsy, and ataxia [5]. To evaluate the performance

729 of ASpediaFI for identifying biologically relevant pathways, we performed gene set

730 enrichment analysis (GSEA) on gene nodes with permutation $P$-values less than 0.05 using

731 DAVID v6.8 [55]. Our GSEA result was compared with previously identified two gene sets:

732 blue module and DAS [5]. The blue module comprising 737 genes is a subnetwork identified

733 by WGCNA using gene expression profiles; DAS contained 603 differentially spliced genes

734 detected by DESeq [56]. We also explored the sequential features of our AS events retrieved

735 from the ASpedia database. 
738 To compare the performance of ASpediaFI against other widely used DAS detection tools,

739 we extended case study 1 using the SF3B1-associated MDS dataset. In addition to rMATS

740 v4.0.2, we applied MISO v0.5.4 and SUPPA2 v2.3 to the same MDS RNA-Seq dataset [7-

$7419,13]$. We customized settings for DAS analysis to reflect the characteristics of each tool.

742 rMATS analysis results were collected from case study 1 and additional cutoffs $(|\mathrm{dPSI}|>0.1$

743 and FDR < 5\%) were applied. For MISO, as only pairwise comparisons are allowed in DAS

744 analysis, we merged BAM files for multiple samples per condition (SF3B1 MUT: 28 cases

745 and WT: 56 controls). DAS analysis was performed using the pooled version of BAM files,

746 and other parameters were used in default settings. We, therefore, filtered the resultant DAS

747 events with more stringent minimal coverage and Bayes factor (BF) than default values (BF $\geq$

74820 , the sum of inclusion and exclusion reads $\geq 300$, at least 30 inclusion and exclusion reads),

749 and eliminated AS events by the same cutoff $(|\mathrm{dPSI}| \leq 0.1)$ with rMATS. For SUPPA2, to

750 obtain PSI profiles, we quantified transcript expression in TPM units using RSEM v1.3.0.

751 Next, we executed the embedded modules psiPerEvent to generate PSI profiles and diffSplice

752 to detect DAS events using default options. The same thresholds with rMATS were also

753 applied to select final DAS events derived from SUPPA2. To evaluate the performance of the

754 four methods, we tested gene set enrichment of HM pathway referring to the top-ranking

755 results in case study 1 and previously published studies [4,13,23-26]. To test the enrichment

756 of DAS events for each tool, we converted DAS events to gene symbols and computed

757 Fisher's exact test $P$-values and F1 scores for HM and expansion pathway gene sets.

758 Performance benchmark using simulated datasets

759 To evaluate the ability to detect functionally-enriched DAS of ASpediaFI, we generated a

760 simulation dataset close to the actual MDS patient RNA-Seq dataset. To define the ground 
761 truth AS gene set for simulation, we intended to select AS genes that are highly likely to

762 occur for the real MDS samples instead of randomly chosen genes. Therefore we investigated

763 gene sets using three different methods. We applied the same running options for rMATS,

764 MISO, and SUPPA2 as with previous evaluations using MDS samples. To identify more

765 DAS genes on intersection set, we imposed relatively less stringent cutoffs: $\mid$ dPSI $\mid>0.025$,

766 FDR $<10 \%$ for rMATS and SUPPA2. For MISO, additional strict thresholds were applied to

767 balance the number of DAS events with the other two methods (BF $\geq 800$, the sum of

768 inclusion and exclusion reads $\geq 700)$, as well as the same relaxed cutoff $(|\mathrm{dPSI}|>0.025)$.

769 Finally, the resulting set of DAS genes overlapping between the three tools was assigned as

770 our ground truth for the simulated dataset.

771 Next, we generated 20 replicates per condition (SF3B1 MUT and WT) via Flux Simulator

772 [57], executing scripts from the previous simulation study [12]. To simulate realistic RNA-

773 Seq reads, we referred to the real RNA-Seq samples of MDS patients with SF3B1 MUT and

774 WT. Transcript counts were sampled from a negative binomial distribution with mean and

775 variance estimated for MUT and WT conditions of the original MDS BAM files. For the

776 deliberately chosen true DAS genes, we set relative isoform abundances such that the last

777 isoform took a pre-determined proportion ( 0.8 for MUT and 0.2 for WT), while others

778 equally shared the rest. Isoform-level abundances of other genes were drawn from a uniform

779 distribution. The simulated RNA-Seq reads for each replicate with mean base coverage of 65

780 were then mapped to the GRCh38 genome along with the GENCODE v31 gene model, using

781 STAR v2.5.1b. Additionally, to evaluate the effect of sample size, 10 and 5 replicates per

782 condition were randomly chosen from the full simulated dataset. We performed DAS tests

783 using the simulation dataset for ASpediaFI and three other methods. ASpediaFI was run with

784 the following options: restart $=0.7$, num.folds $=5$, num.feats $=500$, low.expr $=1$, low.var $=$ 
785 NULL, prop.na $=0.05$, prop.extreme $=1$. For each simulated datasets of different sizes $(n=20$,

78610,5 replicates per condition), the cor.threshold option was adjusted by the number of

787 detected AS event nodes (0.4, 0.5, 0.8, respectively). The three tools were applied using

788 options previously described. As the genome-wide ranking results were compared, additional

789 filtering by dPSI was excluded.

790 To evaluate the accuracy of the four methods, we generated receiver operating characteristic

791 (ROC) curve and computed the area under the curve (AUC-ROC) metric, using R PRROC

792 package [58]. We also calculated the area under the precision-recall curve (AUC-PR) metric.

793 Ranking of AS events were computed based on measures of $1-$ adjusted $P$ values for rMATS

794 and SUPPA2, BF for MISO, and stat-P for ASpediaFI S1 and S2 were provided. Moreover,

795 to assess the effect of sample size, we computed AUC-ROC and AUC-PR metrics using the

796 simulated datasets of randomly-chosen smaller sample sizes $(n=10,5$ replicates per

797 condition).

798 For further performance evaluation, we investigated pathway enrichment, and evaluated

799 whether the four methods maintained their ability to identify biologically-relevant AS events

800 using the simulated dataset. Based on previous studies and case study 1, we assumed that the

801 HM pathway is dysregulated in MDS patients with SF3B1 mutation [4,13,23-26]. As our

802 simulation dataset was derived from the actual MDS patient sample analysis result, we

803 investigated the pathway status similar to the previously described process. To confirm

804 GSEA consistency between DAS and DEG, we applied GAGE to perform GSEA using gene

805 expression profiles for hallmark pathways [59]. Next, for the DAS enrichment test, we

806 extracted an equal number (top 500) of most significant DAS events for each tool after

807 filtering out by $|\mathrm{dPSI}| \leq 0.1$. Finally, we assessed the enrichment of AS event sets for the four 
808 methods by conducting Fisher's exact test and computing the $F_{1}$ score from HM and

809 expansion sets.

810 Abbreviations

811 AS: Alternative splicing

812 DRaWR: Discriminative random walk with restart

813 DAS: Differential alternative splicing

814 DEG: Differentially expressed genes

815 EMT: Epithelial-to-mesenchymal

816 GSEA: Gene set enrichment analysis

817 PSI: Percent spliced in

818 SF: Splicing factor

819 Stat-P: Stationary probability

820

821 Declarations

822 Ethics approval and consent to participate

823 Ethics approval was not applicable for this study.

824 Competing interests

825 The authors declare that they have no competing interests. 


\section{Availability of data and materials}

827 Datasets used in this manuscript are accessible at GEO (accession: GSE114922 and

828 GSE36710) and GDC (TCGA STAD RNA-Seq level3). ASpediaFI is supported as an R

829 package open source program. The tool, user manual and case study are publicly available at

830 Bioconductor (https://bioconductor.org/packages/ASpediaFI).

831 Authors' contribution

832 Conceptualization, supervision and funding acquisition, C.P.; algorithm implementation and

833 analysis, D.Y., K.L., and D.H.; evaluation, D.Y. and K.L; writing, review, and editing, D.Y.,

834 K.L. S.Y.C., and C.P.

\section{Funding}

836 This work was supported by National Research Foundation of Korea grant funded by the

837 Korea government (NRF-2019R1A2C1003401); National Cancer Center Grant (NCC-

838 1910040).

839 


\section{REFERENCES}

841 1. Yang X, Coulombe-Huntington J, Kang S, Sheynkman GM, Hao T, Richardson A, et al.

842 Widespread Expansion of Protein Interaction Capabilities by Alternative Splicing. Cell.

$8432016 ; 164: 805-17$.

844 2. Saha A, Kim Y, Gewirtz ADH, Jo B, Gao C, McDowell IC, et al. Co-expression networks

845 reveal the tissue-specific regulation of transcription and splicing. Genome Res.

$846 \quad 2017 ; 27: 1843-58$.

847 3. Salomonis N, Schlieve CR, Pereira L, Wahlquist C, Colas A, Zambon AC, et al.

848 Alternative splicing regulates mouse embryonic stem cell pluripotency and differentiation.

849 Proc Natl Acad Sci U S A. 2010;107:10514-9.

850 4. Dolatshad H, Pellagatti A, Liberante FG, Llorian M, Repapi E, Steeples V, et al. Cryptic

851 splicing events in the iron transporter $\mathrm{ABCB} 7$ and other key target genes in SF3B1-mutant

852 myelodysplastic syndromes. Leukemia. 2016;30:2322-31.

853 5. Fogel BL, Wexler E, Wahnich A, Friedrich T, Vijayendran C, Gao F, et al. RBFOX1

854 regulates both splicing and transcriptional networks in human neuronal development. Hum

855 Mol Genet. 2012;21:4171-86.

856 6. Seiler M, Peng S, Agrawal AA, Palacino J, Teng T, Zhu P, et al. Somatic Mutational

857 Landscape of Splicing Factor Genes and Their Functional Consequences across 33 Cancer

858 Types. Cell Rep. 2018;23:282-296.e4.

859 7. Shen S, Park JW, Lu Z, Lin L, Henry MD, Wu YN, et al. rMATS: Robust and flexible

860 detection of differential alternative splicing from replicate RNA-Seq data. Proc Natl Acad Sci.

861 2014;111:E5593-601. 
862 8. Trincado JL, Entizne JC, Hysenaj G, Singh B, Skalic M, Elliott DJ, et al. SUPPA2: fast,

863 accurate, and uncertainty-aware differential splicing analysis across multiple conditions.

864 Genome Biol. 2018;19:40.

865 9. Katz Y, Wang ET, Airoldi EM, Burge CB. Analysis and design of RNA sequencing

866 experiments for identifying isoform regulation. Nat Methods. 2010;7:1009-15.

867 10. Wang Q, Rio DC. JUM is a computational method for comprehensive annotation-free

868 analysis of alternative pre-mRNA splicing patterns. Proc Natl Acad Sci. 2018;115:E8181-90.

869 11. Saraiva-Agostinho N, Barbosa-Morais NL. psichomics: graphical application for

870 alternative splicing quantification and analysis. Nucleic Acids Res. 2019;47:e7-e7.

871 12. Liu R, Loraine AE, Dickerson JA. Comparisons of computational methods for differential

872 alternative splicing detection using RNA-seq in plant systems. BMC Bioinformatics.

$873 \quad 2014 ; 15: 364$.

874 13. Pellagatti A, Armstrong RN, Steeples V, Sharma E, Repapi E, Singh S, et al. Impact of

875 spliceosome mutations on RNA splicing in myelodysplasia: dysregulated genes/pathways and

876 clinical associations. Blood. 2018;132:1225-40.

877 14. Irimia M, Weatheritt RJ, Ellis JD, Parikshak NN, Gonatopoulos-Pournatzis T, Babor M,

878 et al. A highly conserved program of neuronal microexons is misregulated in autistic brains.

879 Cell. 2014;159:1511-23.

880 15. Lee SCW, Abdel-Wahab O. Therapeutic targeting of splicing in cancer. Nat. Med. 2016.

881 p. 976-86. 
882 16. Warzecha CC, Sato TK, Nabet B, Hogenesch JB, Carstens RP. ESRP1 and ESRP2 Are

883 Epithelial Cell-Type-Specific Regulators of FGFR2 Splicing. Mol Cell. 2009;33:591-601.

884 17. Wang B-D, Ceniccola K, Hwang S, Andrawis R, Horvath A, Freedman JA, et al.

885 Alternative splicing promotes tumour aggressiveness and drug resistance in African

886 American prostate cancer. Nat Commun. 2017;8:15921.

887 18. Liberzon A, Birger C, Thorvaldsdóttir H, Ghandi M, Mesirov JP, Tamayo P. The

888 Molecular Signatures Database Hallmark Gene Set Collection. Cell Syst. 2015;1:417-25.

889 19. Croft D, O’Kelly G, Wu G, Haw R, Gillespie M, Matthews L, et al. Reactome: a database

890 of reactions, pathways and biological processes. Nucleic Acids Res. 2011;39:D691-7.

891 20. Blatti C, Sinha S. Characterizing gene sets using discriminative random walks with restart

892 on heterogeneous biological networks. Bioinformatics. 2016;32:2167-75.

893 21. Valdeolivas A, Tichit L, Navarro C, Perrin S, Odelin G, Levy N, et al. Random walk with

894 restart on multiplex and heterogeneous biological networks. Bioinformatics. 2019;35:497-

895505.

896 22. Hyung D, Kim J, Cho SY, Park C. ASpedia $\square$ : a comprehensive encyclopedia of human

897 alternative splicing. Nucleic Acids Res. 2018;46:58-63.

898 23. Pellagatti A, Cazzola M, Giagounidis AAN, Malcovati L, Della Porta MG, Killick S, et al.

899 Gene expression profiles of CD34+ cells in myelodysplastic syndromes: Involvement of

900 interferon-stimulated genes and correlation to FAB subtype and karyotype. Blood.

$901 \quad 2006 ; 108: 337-45$. 
902 24. Dolatshad H, Pellagatti A, Fernandez-Mercado M, Yip BH, Malcovati L, Attwood M, et

903 al. Disruption of SF3B1 results in deregulated expression and splicing of key genes and

904 pathways in myelodysplastic syndrome hematopoietic stem and progenitor cells. Leukemia.

$905 \quad 2015 ; 29: 1092-103$.

906 25. Conte S, Katayama S, Vesterlund L, Karimi M, Dimitriou M, Jansson M, et al. Aberrant

907 splicing of genes involved in haemoglobin synthesis and impaired terminal erythroid

908 maturation in SF3B1 mutated refractory anaemia with ring sideroblasts. Br J Haematol.

$909 \quad 2015 ; 171: 478-90$.

910 26. Shiozawa Y, Malcovati L, Gallì A, Sato-Otsubo A, Kataoka K, Sato Y, et al. Aberrant

911 splicing and defective mRNA production induced by somatic spliceosome mutations in

912 myelodysplasia. Nat Commun. 2018;9:3649.

913 27. de Oliveira RM, Vicente Miranda H, Francelle L, Pinho R, Szegö ÉM, Martinho R, et al.

914 The mechanism of sirtuin 2-mediated exacerbation of alpha-synuclein toxicity in models of

915 Parkinson disease. PLoS Biol. 2017;15.

916 28. Nakamura K, Kageyama S, Yue S, Huang J, Fujii T, Ke B, et al. Heme oxygenase-1

917 regulates sirtuin-1-autophagy pathway in liver transplantation: From mouse to human. Am J

918 Transplant. 2018;18:1110-21.

919 29. Shao J, Grammatikakis N, Scroggins BT, Uma S, Huang W, Chen JJ, et al. Hsp90

920 regulates p50cdc37 function during the biogensis of the active conformation of the heme-

921 regulated eIF2 $\alpha$ kinase. J Biol Chem. 2001;276:206-14. 
922 30. Warzecha CC, Shen S, Xing Y, Carstens RP, Warzecha CC, Shen S, et al. The epithelial

923 splicing factors ESRP1 and ESRP2 positively and negatively regulate diverse types of

924 alternative splicing events Claude. RNA Biol. 2009;6:546-62.

925 31. Shapiro IM, Cheng AW, Flytzanis NC, Balsamo M, Condeelis JS, Oktay MH, et al. An

926 emt-driven alternative splicing program occurs in human breast cancer and modulates cellular

927 phenotype. PLoS Genet. 2011;7:e1002218.

928 32. Hänzelmann S, Castelo R, Guinney J. Open Access GSVA $\square$ : gene set variation analysis

929 for microarray and RNA-Seq data. BMC Bioinformatics. 2013;14.

930 33. Yang Y, Park JW, Bebee TW, Warzecha CC, Guo Y, Shang X, et al. Determination of a

931 Comprehensive Alternative Splicing Regulatory Network and Combinatorial Regulation by

932 Key Factors during the Epithelial-to-Mesenchymal Transition. Mol Cell Biol. 2016;36:1704-

93319.

934 34. Warzecha CC, Jiang P, Amirikian K, Dittmar KA, Lu H, Shen S, et al. An ESRP-

935 regulated splicing programme is abrogated during the epithelial-mesenchymal transition.

936 EMBO J. 2010;29:3286-300.

937 35. Dittmar KA, Jiang P, Park JW, Amirikian K, Wan J, Shen S, et al. Genome-wide

938 determination of a broad ESRP-regulated posttranscriptional network by high-throughput

939 sequencing. Mol Cell Biol. 2012;32:1468-82.

940 36. Wieczorek K, Wiktorska M, Sacewicz-Hofman I, Boncela J, Lewiński A, Kowalska MA,

941 et al. Filamin A upregulation correlates with Snail-induced epithelial to mesenchymal

942 transition (EMT) and cell adhesion but its inhibition increases the migration of colon

943 adenocarcinoma HT29 cells. Exp Cell Res. 2017;359:163-70. 
944 37. Di Modugno F, Iapicca P, Boudreau A, Mottolese M, Terrenato I, Perracchio L, et al.

945 Splicing program of human MENA produces a previously undescribed isoform associated

946 with invasive, mesenchymal-like breast tumors. Proc Natl Acad Sci U S A. 2012;109:19280-

9475.

948 38. Weise A, Bruser K, Elfert S, Wallmen B, Wittel Y, Wöhrle S, et al. Alternative splicing

949 of Tcf712 transcripts generates protein variants with differential promoter-binding and

950 transcriptional activation properties at Wnt/beta-catenin targets. Nucleic Acids Res.

$951 \quad 2010 ; 38: 1964-81$.

952 39. Cristescu R, Lee J, Nebozhyn M, Kim KM, Ting JC, Wong SS, et al. Molecular analysis

953 of gastric cancer identifies subtypes associated with distinct clinical outcomes. Nat Med.

$954 \quad 2015 ; 21: 449-56$.

955 40. Papaemmanuil E, Cazzola M, Boultwood J, Malcovati L, Vyas P, Bowen D, et al.

956 Somatic SF3B1 mutation in myelodysplasia with ring sideroblasts. N Engl J Med.

$957 \quad 2011 ; 365: 1384-95$.

958 41. Bader GD, Betel D, Hogue CW V. BIND: the Biomolecular Interaction Network

959 Database. Nucleic Acids Res. 2003;31:248-50.

960 42. Xenarios I, Rice DW, Salwinski L, Baron MK, Marcotte EM, Eisenberg D. DIP: the

961 database of interacting proteins. Nucleic Acids Res. 2000;28:289-91.

962 43. Keshava Prasad TS, Goel R, Kandasamy K, Keerthikumar S, Kumar S, Mathivanan S, et

963 al. Human Protein Reference Database--2009 update. Nucleic Acids Res. 2009;37:D767-72.

964 44. Kanehisa M, Sato Y, Kawashima M, Furumichi M, Tanabe M. KEGG as a reference

965 resource for gene and protein annotation. Nucleic Acids Res. 2016;44:D457-62. 
966 45. de Winter JCF, Gosling SD, Potter J. Comparing the pearson and spearman correlation

967 coefficients across distributions and sample sizes: A tutorial using simulations and empirical

968 data. Psychol Methods. 2016;21:273-90.

969 46. Zhu L, Su F, Xu YC, Zou Q. BBA - Molecular Basis of Disease Network-based method

970 for mining novel HPV infection related genes using random walk with restart algorithm.

$971 \quad$ BBA - Mol Basis Dis. 2018;1864:2376-83.

972 47. Dobin A, Davis CA, Schlesinger F, Drenkow J, Zaleski C, Jha S, et al. STAR: ultrafast

973 universal RNA-seq aligner. Bioinformatics. 2013;29:15-21.

974 48. Li B, Dewey CN. RSEM: accurate transcript quantification from RNA-Seq data with or

975 without a reference genome. BMC Bioinformatics. 2011;12:323.

976 49. Ritchie ME, Phipson B, Wu D, Hu Y, Law CW, Shi W, et al. limma powers differential

977 expression analyses for RNA-sequencing and microarray studies. 2015;43.

978 50. Network TCGAR. Comprehensive molecular characterization of gastric adenocarcinoma.

979 Nature. 2014;513:202-9.

980 51. Sebestyén E, Singh B, Miñana B, Pagès A, Mateo F, Pujana MA, et al. Large-scale

981 analysis of genome and transcriptome alterations in multiple tumors unveils novel cancer-

982 relevant splicing networks. Genome Res. 2016;26:732-44.

983 52. Zhang C, Zhang Z, Castle J, Sun S, Johnson J, Krainer AR, et al. Defining the regulatory

984 network of the tissue-specific splicing factors Fox-1 and Fox-2. Genes Dev. 2008;22:2550-

98563. 
986 53. Yeo GW, Coufal NG, Liang TY, Peng GE, Fu XD, Gage FH. An RNA code for the

987 FOX2 splicing regulator revealed by mapping RNA-protein interactions in stem cells. Nat.

988 Struct. Mol. Biol. 2009. p. 130-7.

989 54. Voineagu I, Wang X, Johnston P, Lowe JK, Tian Y, Horvath S, et al. Transcriptomic

990 analysis of autistic brain reveals convergent molecular pathology. Nature. 2011;474:380-6.

991 55. Huang DW, Sherman BT, Lempicki RA. Systematic and integrative analysis of large

992 gene lists using DAVID bioinformatics resources. Nat Protoc. 2009;4:44-57.

993 56. Anders S, Huber W. Differential expression analysis for sequence count data. Genome

994 Biol. 2010;11.

995 57. Griebel T, Zacher B, Ribeca P, Raineri E, Lacroix V, Guigó R, et al. Modelling and

996 simulating generic RNA-Seq experiments with the flux simulator. Nucleic Acids Res.

$997 \quad 2012 ; 40: 10073-83$.

998 58. Grau J, Grosse I, Keilwagen J. PRROC: computing and visualizing precision-recall and

999 receiver operating characteristic curves in R. Bioinformatics. 2015;31:2595-7.

1000 59. Luo W, Friedman MS, Shedden K, Hankenson KD, Woolf PJ. GAGE: Generally

1001 applicable gene set enrichment for pathway analysis. BMC Bioinformatics. 2009;10:161.

1002

1003 


\section{TABLES AND FIGURES}

1005 Figure 1. ASpediaFI workflow and DRaWR algorithm to identify AS interaction subnetwork.

1006 A) ASpediaFI establishes a heterogeneous network using gene interaction, gene-AS

1007 correlation, and gene-pathway association data. AS events are annotated from a GTF file, and

1008 PSI calculation using BAM files is also embedded. Public gene sets are referred for gene-

1009 pathway associations. B) A heterogeneous network is composed of genes and its feature

1010 nodes, AS events and pathways. Gene-gene and gene-AS interaction edges are weighted by

1011 correlations of gene expression and PSI values. Next, all edge weights are normalized for

1012 each type of feature interaction and each column. The first stage RWR explores a

1013 heterogeneous network starting from nodes in a query gene set (blue nodes). The second

1014 stage RWR finalizes scores within a query-specific subnetwork derived from the first stage.

1015 ASpediaFI additionally computes permutation $P$-values of the gene nodes to eliminate the

1016 effect of the background gene set.

1017 Figure 2. MDS patient RNA-Seq dataset analysis to identify AS events and pathways

1018 regulated by SF3B1, SRSF2, and U2AF1 mutations. A) Percentages of five AS types

1019 identified by ASpediaFI for three SF MUT cases. B) Heatmap of the top 15 pathways ranked

1020 by stat-Ps. C) PCA plot derived from PSI profiles of SF3B1 MUT-associated 281 events.

1021 PC1 (x-axis) and PC2 (y-axis) indicate principal component 1 and 2. D) Two barplots of AS

1022 event enrichment comparison in HM pathway gene set for three conditions: ASpediaFI,

1023 rMATS Cond1, and Cond2. One is negative log-scale $P$-values of Fisher's exact method and

1024 other's Jaccard indices. E) A Venn diagram of genes related to SF3B1-associated AS events

1025 identified by ASpediaFI and rMATS (Cond2) compared with the HM expansion set

1026 containing both HM pathway gene set and interacting novel gene set. For testing enrichment

1027 with HM expansion, $P$-values for ASpediaFI and rMATS were calculated by Fisher's exact 
1028 test. In two exclusive intersections of ASpediaFI $(n=22)$ and rMATS $(n=8)$, ASpediaFI

1029 detected more events $(n=10)$ in the expansion set than rMATS $(n=5)$ as well as total events in

1030 two exclusive intersections. F) Percentage barplots of AS events to contain four functional

1031 sequence features, protein domain, NMD, PTM, and PPI. It was also compared with rMATS

1032 Cond1 and Cond2.

1033 Figure 3. AS events associated with the EMT pathway regulated by splicing factor ESRP1. A)

1034 PCA scatter plot using PSI profiles using 293 AS events. B) Percentage pie chart of five AS

1035 types. C) Pathway identification comparison between our method and gene expression-based

1036 analysis. Seven pathways in heatmap row were chosen from ASpediaFI pathway ranking, and

1037 columns were ordered by high and low groups. The heatmap demonstrates pathway-level

1038 GSVA scores estimated using gene expression profiles. The barplot on the right layout

1039 presents both our stat-P values (gray) for pathway ranking and log-scaled adjusted $P$-values

1040 (white) of GSVA scores comparing between ESRP1 high and low groups. D) Venn diagram

1041 of ASpediaFI, SUPPA2, and the EMT expansion gene set. $P$-values for two AS sets denote

1042 enrichment with EMT expansion set. E) Status barplots to investigate AS event consistency

1043 identified by ASpediaFI and SUPPA2. Five EMT splicing gene signatures (Yang ESRP1 [33],

1044 Yang EMT [33], Warzecha [34], Dittmar [35], and Shapiro [31]) were collected, and Fisher's

1045 exact test $P$-values and Jaccard indices were calculated. F) Scatter plots between EMT

1046 pathway scores (y-axis) by GSVA and square-root-transformed AS event PSI values (x-axis)

1047 for three AS events, ENAH, FGFR2, and TCG7L2. Correlation coefficients were added to

1048 each plot. Blue dots indicate low group and red dots indicate high group. G) A gene-AS

1049 interaction subnetwork identified by ASpediaFI. Circle nodes denote gene nodes, and

1050 hexagons are AS events. AS event nodes were filled in color by dPSI values. To extract

1051 smaller size EMT-relevant subnetwork for generating plot, we eliminated gene nodes 
1052 belonging to the EMT expansion set with $\log 2$ fold change $<2$ and AS nodes of $|\mathrm{dPSI}|<$

1053 0.25. Multiple edges of one AS node were trimmed except the one with the maximum score.

1054 The dotted line ellipse indicates the interactions of three spliced genes (Figure 3F).

1055 Figure 4. Analysis of the RBFOX1 knockdown RNA-Seq dataset. A) A pie chart showing 1056 the proportion of 5 AS event types. B) Jaccard index barplots between our result and splicing 1057 gene signatures collected from a previous study [5]. Three relevant RBFOX1-associated 1058 splicing gene sets were overlapped with three controls. C) Dot plot for percentile ranks of GO 1059 terms (row) from gene sets (column) by three different methods, our genes extracted by 1060 permutation $P$-values (FI), neuronal development genes identified by WGCNA referring gene 1061 expression (Blue Module), and differentially spliced genes (DAS). The last two gene sets 1062 were derived from the previous study result. D) RBFOX1-associated subnetwork that 1063 ASpediaFI identified. To extract a smaller size subnetwork, we eliminated gene nodes 1064 belonging to neuron differentiation set with $\log 2$ fold change $<0.25$ and AS nodes of $\mid$ dPSI $\mid$ 1065 (<0.15). E) Exonic structure of exon 18 skipping (red) and protein domains of ROBO1.

1066 Figure 5. Performance evaluation of ASpediaFI and comparison with three other methods 1067 (MISO, rMATS, and SUPPA2) (A) Barplots of Fishers' exact test $P$-values and $F_{1}$ scores to 1068 test pathway enrichment for both HM and expansion sets. Enrichment was tested from two 1069 pathway gene sets, and AS event gene sets identified from four methods. (B) Venn diagram 1070 of DAS genes among three methods analyzing case study 1 MDS dataset. The intersecting 1071 DAS genes $(n=125)$ among all three methods serve as the ground truth for the simulated 1072 dataset. (C) ROC curves to evaluate the accuracy of four methods detecting DAS from a 1073 simulated dataset. ROC curves for each method illustrate true-positive rate (y-axis) against 1074 false-positive rate (x-axis). AUC values are described for each method. The dotted diagonal 
1075 line corresponds to a ROC curve when DAS predictions are randomly guessed (AUC $=0.5)$.

1076 (D) Barplots of AUC-ROC and AUC-PR for the evaluation of sample size effect ( $\mathrm{n}=20,10$,

10775 replicates per condition). Bar colors indicate the same method as in Figure 5C. (E) GSEA

$1078 P$-value barplot of highly ranked hallmark pathway from the simulated dataset that we imitate

1079 SF3B1 MUT and WT. HM pathway is detected on top. (F) Barplots of Fishers' exact test $P$ -

1080 values and $F_{1}$ scores to test pathway enrichment for both HM and expansion sets. AS event

1081 sets were extracted from the simulated data analysis using four methods. 
Figure 1

A

Network Construction
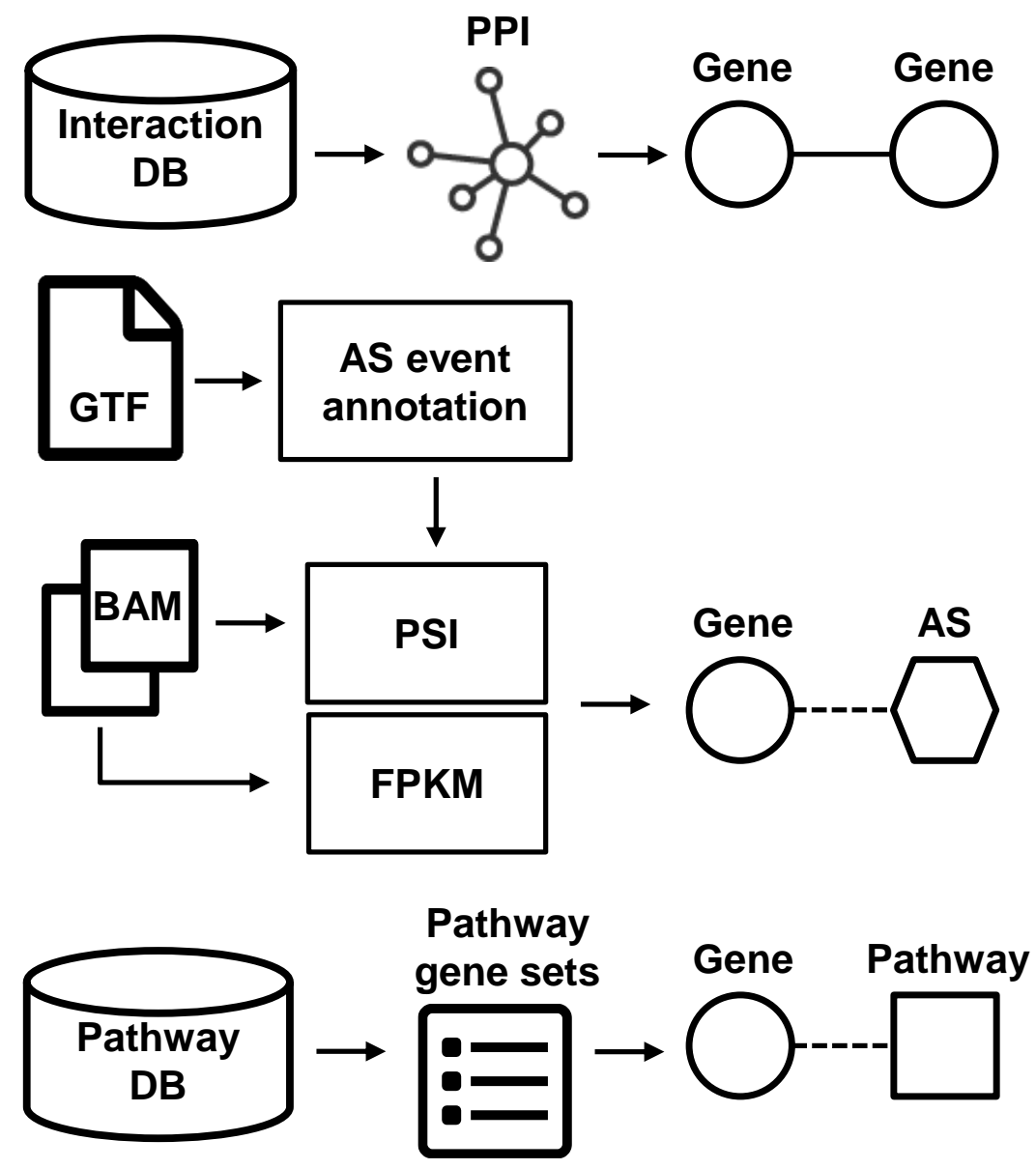

B

\section{DRaWR}

First-stage RWR

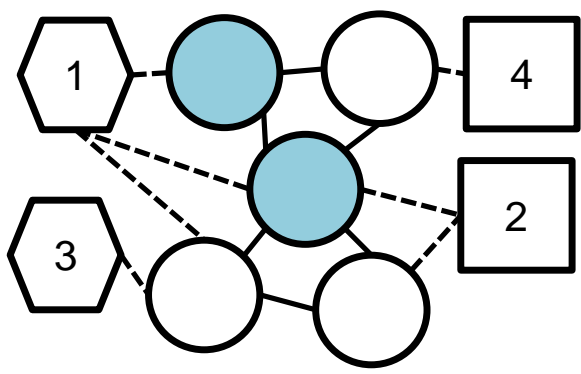

Second-stage RWR

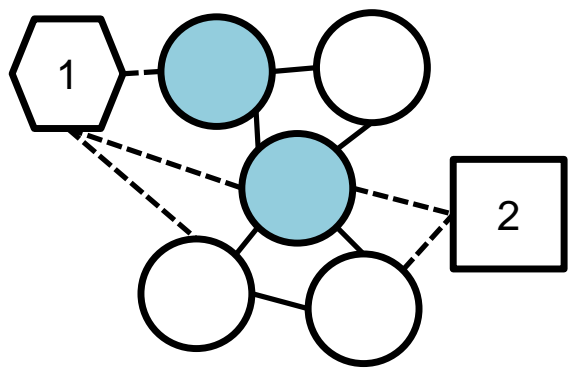

\section{Permutation Test}

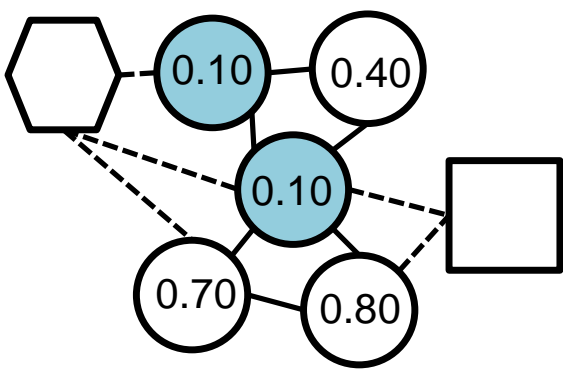


Figure 3

A

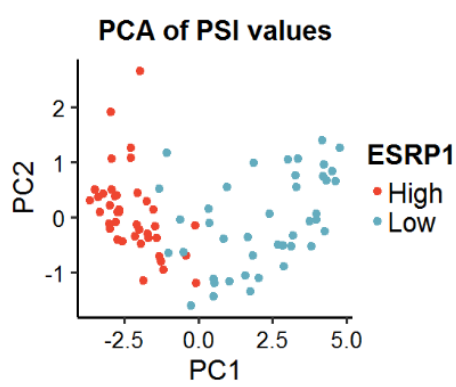

B

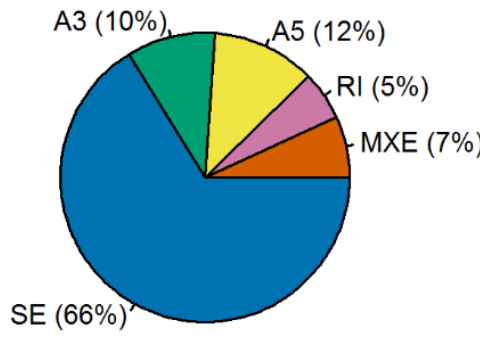

E
EMT (1)

ASpediaFI $(\mathrm{P}=0.003)$
SUPPA2

$(P=0.003)$

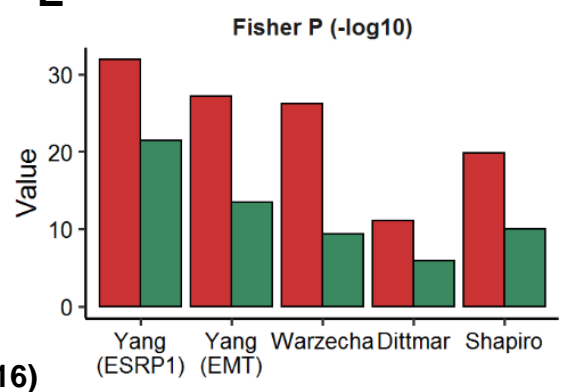

stat-P

$\begin{array}{lllll}0.0000 & 0.0025 & 0.0050 & 0.0075\end{array}$ EMT Myogenesis Apical Junct. KRAS Sign. Up Inflammatory Resp. IL2 STAT5 Sign. UV Resp. Down

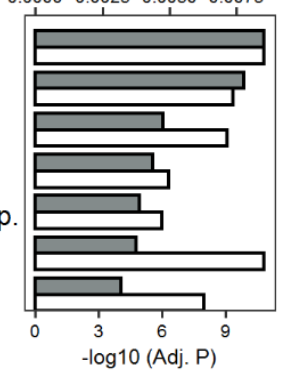

Jaccard Index

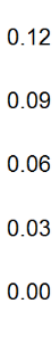

EMT (7)
Expansion (16)

Expansion (16)

ASpediaFI $\square$ SUPPA2

G
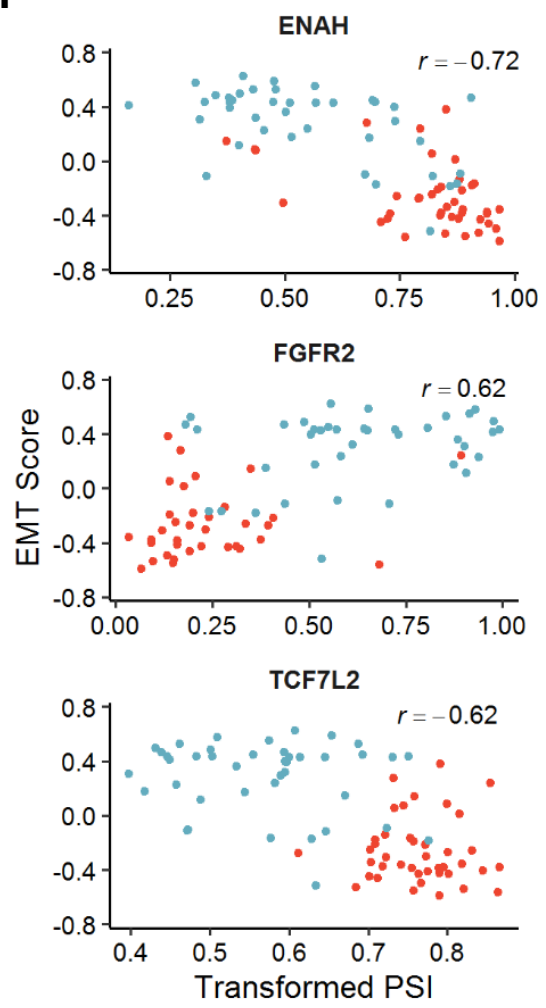

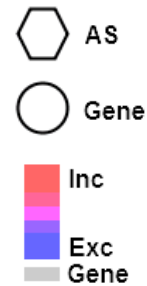

Gene

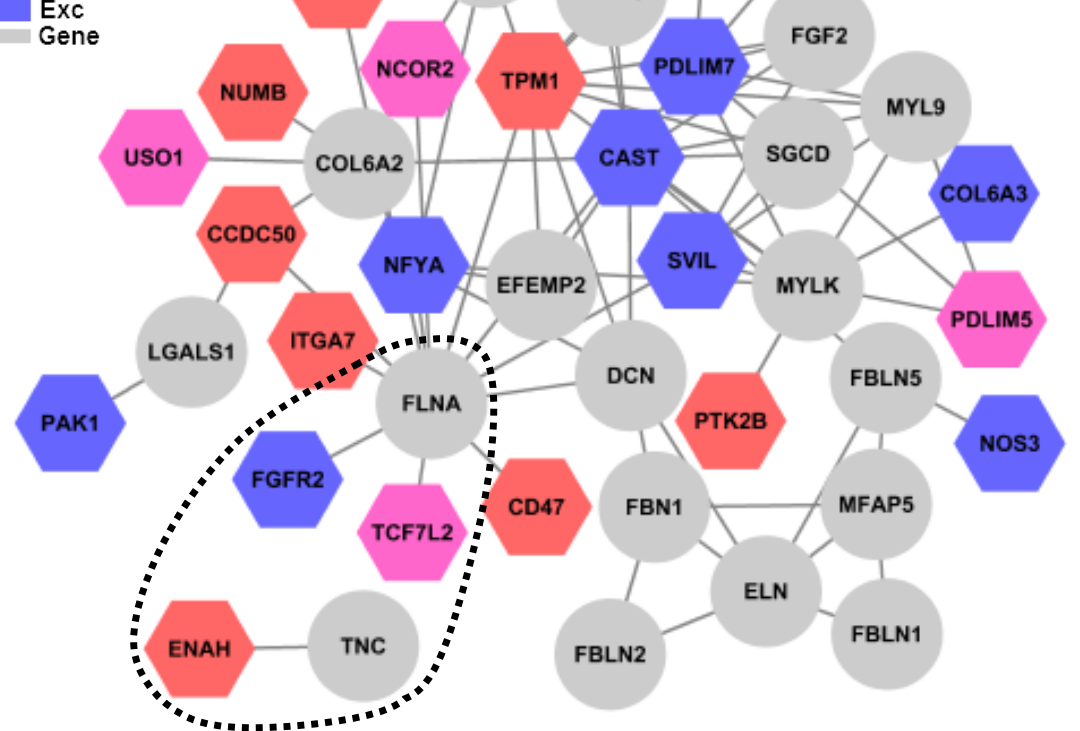




\section{Figure 4}

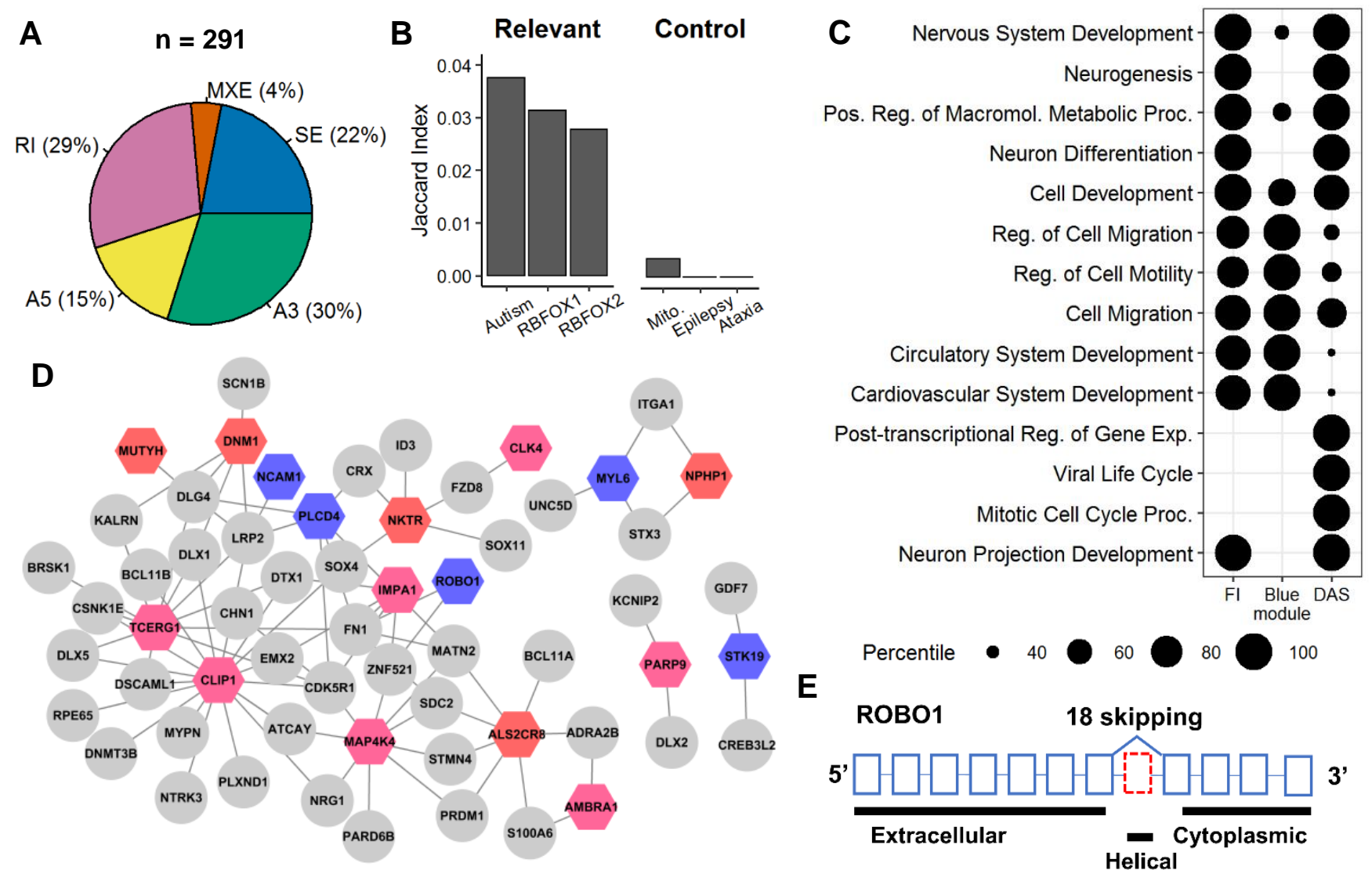




\section{Figure 5}

A

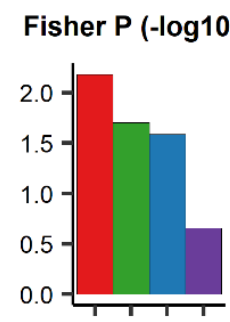

HM

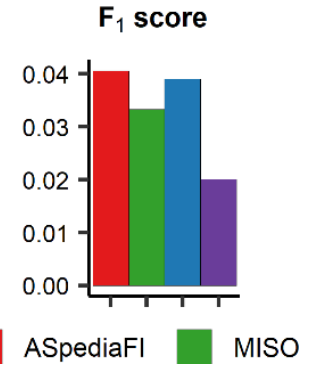

HM expansion

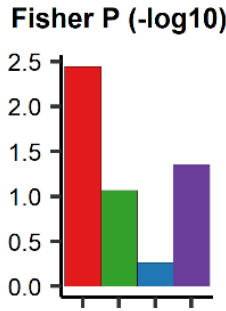

rMATS

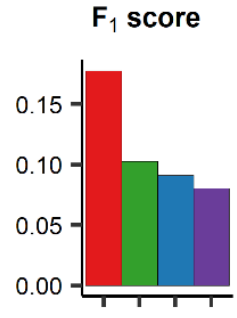

SUPPA2
B

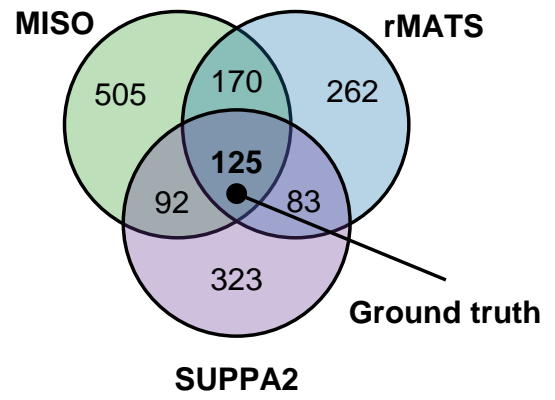

C

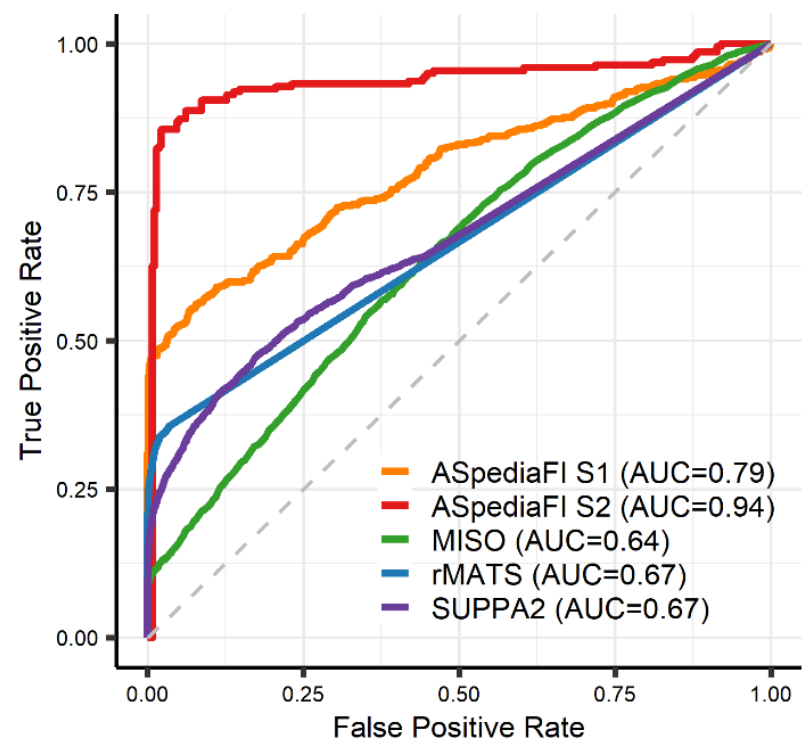

E

Heme Metabolism Allograft Rejection E2F Targets Myc Targets Hypoxia Interferron AlphaDNA Repair MTORC1 Sign. Reactive Oxigen Glycolysis

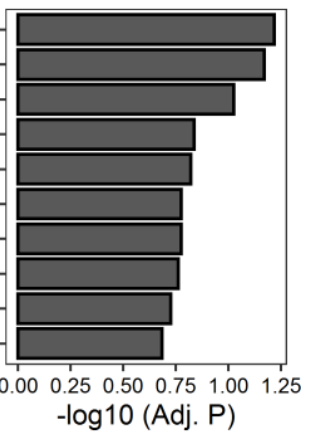

F

Fisher P (-log10)

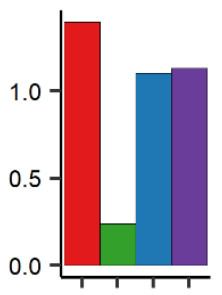

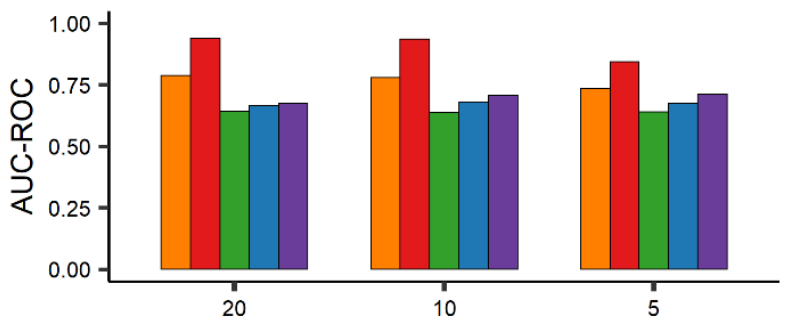

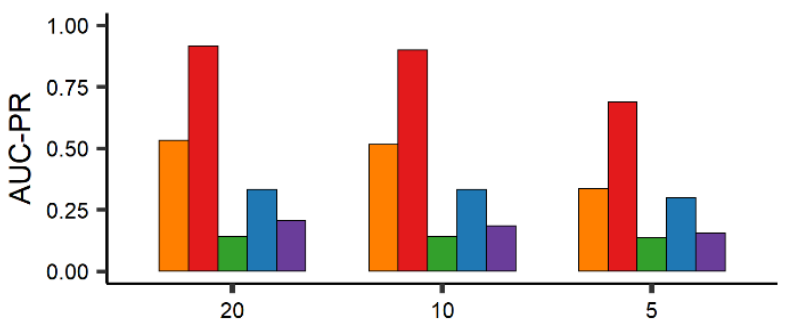

Sample size

HM

$\mathbf{F}_{1}$ score

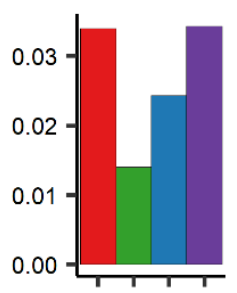

HM expansion

Fisher P (-log10)

$F_{1}$ score
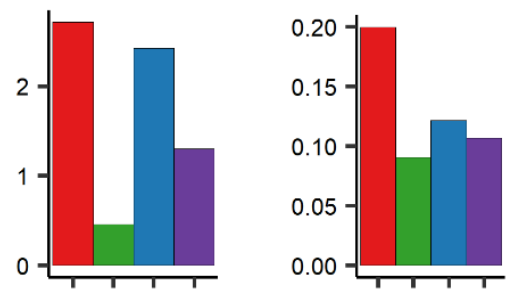